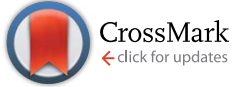

Cite this: RSC Adv., 2017, 7, 15596

Received 21st November 2016 Accepted 21st February 2017

DOI: 10.1039/c6ra27070k

rsc.li/rsc-advances

\section{Influence of laser nanostructured diamond tools on the cutting behavior of silicon by molecular dynamics simulation}

\begin{abstract}
Houfu Dai, ${ }^{\text {abc }}$ Genyu Chen, ${ }^{\text {bc }}$ Shaobo Li, ${ }^{a}$ Qihong Fang ${ }^{b}$ and Bang Hu ${ }^{\text {bc }}$
In this study, a series of large-scale molecular dynamics simulations have been performed to study the nanometric cutting of single crystal silicon with a laser-fabricated nanostructured diamond tool. The material removal behavior of the workpiece using a structured diamond tool cutting is studied. The effects of groove direction, depth, width, factor, and shape on material deformation are carefully investigated by analyzing normal stresses, shear stress, von Mises stress, hydrostatic stress, phase transformation, cutting temperature, cutting force and friction coefficients. Simulation results show that a cutting tool groove orientation of $60^{\circ}$ produces a smaller cutting force, less cutting heat, more beta-silicon phase, and less von Mises stress and hydrostatic stress. Moreover, tools with a smaller groove orientation, groove depth and groove width, and larger groove factor lead to more ductile cutting and an increased material removal rate. However, a cutting tool with a smaller groove width results in more heat during the nanoscale cutting process. In addition, the average temperature of the subsurface increases as groove factor increases, showing that a tool groove accelerates heat dissipation to the subsurface atoms. Furthermore, this $V$-shape groove cutting is shown to improve material removal ability in nanoscale cutting.
\end{abstract}

\section{Introduction}

Microstructured surfaces can produce more dominant functions and features than a smooth surface. For example, a sharp diamond wheel V-tip has been used to fabricate a micropyramid-structured silicon surface. ${ }^{1}$ This micropyramidstructured Si surface has been used to optimize light-trapping properties in solar cells. ${ }^{2}$ A microstructured diamond surface has a lower normal grinding force compared with a traditional electroplate grinding block. ${ }^{3}$ To gain an indepth understanding of the effects of nanostructured surfaces on nanometric cutting, a series of nanostructured diamond tools have been used for the nanoscale cutting of hard and brittle materials, such as silicon.

Monocrystalline silicon is an ideal optical material for weightsensitive infrared applications and other high-added-value products, such as X-ray interferometers and optics, because of its relatively low thermal expansion coefficient, mass density and cost. However, single crystal silicon is a brittle material with a structured surface that is difficult to machine using a mechanical tool because of the rapid tool wear and brittle-mode removal of brittle materials. Furthermore, mechanical machining of microstructured surfaces produces too small an aspect ratio (microarray

\footnotetext{
${ }^{a}$ College of Mechanical Engineering, Guizhou University, Guiyang 550025, China ${ }^{b}$ State Key Laboratory of Advanced Design and Manufacturing for Vehicle Body, Hunan University, Changsha 410082, PR China. E-mail: hdgychen@163.com; Fax: +86 731 88821772; Tel: +8673188821772

'Institute of Laser Technology, Hunan University, Changsha 410082, PR China
}

height/interval) for application in microengineering. ${ }^{4,5}$ In contrast, laser micromachining can obtain microarrays with a much higher aspect ratio on a superhard diamond surface, ${ }^{6}$ and allows the fabrication of arbitrary surface structures. Consequently, laser machining and etching approaches have been introduced. For example, Dhupal et al. ${ }^{7}$ investigated the relationship between processing parameters of pulsed Nd:YAG laser-turning operation in the production of microgrooves on cylindrical ceramic workpieces, coarse-grained diamond grinding wheels were microstructured with a UV nanosecond-pulsed laser proposed by Guo et al. ${ }^{8}$ and Walter et al. ${ }^{9}$ introduced surface microstructuring using a picosecond pulsed laser ablation method to influence the grinding behavior of high-strength bonded CBN grinding wheels. Additionally, with respect to metal and alloy materials, Zhou et al. ${ }^{\mathbf{1 0 , 1 1}}$ measured the thermal diffusivity of solid titanium and $\mathrm{Ti}-$ $6 \mathrm{Al}-4 \mathrm{~V}$ alloy in the solid state using a laser flash method.

To improve the grinding performance of coarse-grained diamond wheels and reduce the grinding force and heat, many studies have investigated the influence of structured tools during the machining process. For example, Oliveira et al. ${ }^{12,13}$ introduced a method for generating regular surface textures by grinding with structured wheels to investigate the effects of structuring on grinding performance. Mohamed et $a{ }^{14}$ reported that the grinding forces of conventional vitrified $\mathrm{Al}_{2} \mathrm{O}_{3}$ wheels could be reduced by $30-60 \%$ using helical grooves on the wheel surface. Guo et al. ${ }^{8}$ showed that subsurface damage depth could be reduced using a microstructured coarse-grained diamond wheel. Subsurface damage depth and surface roughness are both 
reduced by decreasing the interval. Walter et al. ${ }^{9}$ indicated that the geometry of the surface pattern had a much stronger influence on tool grinding characteristics than the nominal active tool surface. Dai et al. ${ }^{15}$ carried out molecular dynamics (MD) analysis, showing that a structured nanoscale tool, when machining brittle material silicon, results in less hydrostatic stress, a lower temperature and smaller cutting force. Some other surface functionalization treatments have been shown to be useful in real applications. For example, Gu et al. ${ }^{16}$ used graphite nanoplatelets (GNPs) to fabricate GNPs/bisphenol-A epoxy resin (GNPs/E-51) nanocomposites with high thermal conductivity via a casting method. The results indicated that the corresponding thermally conductive coefficient of the fGNPs/E-51 nanocomposites was improved to $1.698 \mathrm{~W} \mathrm{~m}^{-1}$ $\mathrm{K}^{-1}$ using $30 \mathrm{wt} \%$ fGNPs, which was eight times higher than that of the original E-51 matrix. Moreover, they synthetically introduced a terminal amine group hyperbranched phenyl polysiloxane $\left(\mathrm{NH}_{2}\right.$-HBPSi) into a bismaleimide/diallylbisphenol A (BMI/DABA) prepolymer to fabricate $\mathrm{NH}_{2}$-HBPSi/BMI/DABA resins. ${ }^{17}$ They also prepared a highly efficient phenylphosphonate-based flame-retardant epoxy resin (FREP) from phenylphosphonic dichloride (PPDCl) and allylamine (AA). ${ }^{18}$

Studies have improved understanding of structured tool cutting, but mainly focused on investigating the influence of structured wheels or tools on the cutting behavior of hard and brittle materials, such as glass, ceramics and silicon. Meanwhile, few studies have systematically investigated the effects of nanostructured tools, including groove direction, depth, width, factor and shape, on the surface integrity of materials undergoing nanoscale cutting. In contrast, in nanometric cutting, experimental work cannot reveal much more than is already known because the experimental timescales are too long to permit direct observation of phenomena occurring at the atomic level, which normally takes place at femtosecond or picosecond timescales. Consequently, MD simulations have been applied to simulate nanoscale cutting processes since the 1980s. ${ }^{19,20}$

In this study, we implemented a series of large-scale MD simulations of the nanometric cutting of single crystal silicon using nanostructured diamond tools, considering the effects of groove direction, depth, width, factor and shape. Furthermore, the dependence of cutting surface morphology on different tool structures is analyzed. We also provide a detailed analysis of the atomistic process of cutting single crystal silicon by studying normal stresses, shear stress, von Mises stress, hydrostatic stress, atomic coordination numbers, common neighbor analysis (CNA), atomic potential energy, cutting temperature, cutting force and friction coefficients.

\section{Simulation method}

A schematic diagram of the nanoscale cutting simulation model with a nanostructured diamond tool is shown in Fig. 1. Considering the high thermal conductivity of the diamond tool and accuracy of the simulation results, the nanostructured diamond tool was modelled as a deformable body. The workpiece
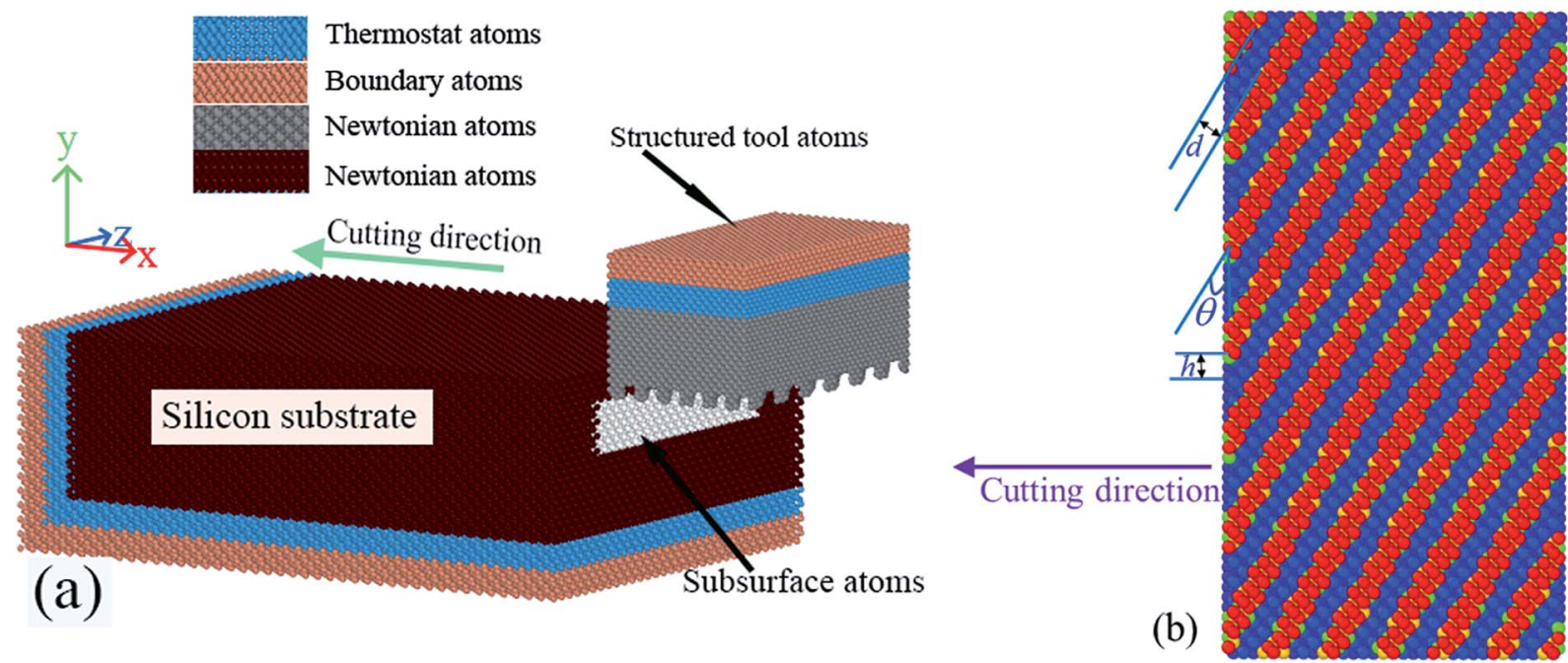

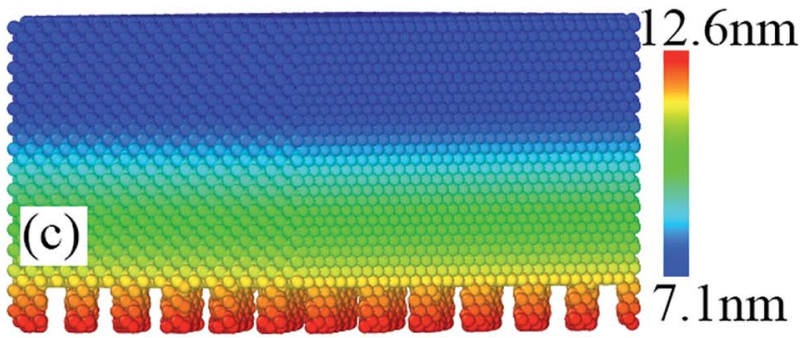

Fig. 1 MD simulation model of nanoscale cutting with a nanostructured diamond tool: (a) three dimensions of the model, (b) surface morphologies of nanostructured diamond tool, and (c) three dimensions of the nanostructured diamond tool. Atoms in (b) and (c) are colored according to height. 
dimensions were $23 \times 8.2 \times 17.4 \mathrm{~nm}^{3}$ in the $x$-, $y$ - and $z$-direction, respectively. All cutting tools were applied along the $\left[\begin{array}{lll}1 & 0 & 0\end{array}\right]$ direction on the $\left(\begin{array}{lll}0 & 1 & 0\end{array}\right)$ silicon surface at a constant cutting speed of $200 \mathrm{~m} \mathrm{~s}^{-1}$. Both the workpiece and tools were divided into three different zones: Newtonian, thermostat and boundary atoms. The boundary atoms were unaffected during simulation and remained fixed in space to reduce boundary effects and maintain lattice symmetry. Atomic motion in the thermostat and Newtonian zones were assumed to follow Newton's second law of motion, which was integrated using the Velocity Verlet algorithm with a time step of 1 fs. Thermostat atoms were set to ensure reasonable outward heat conduction using the direct velocity scaling method. Periodic boundary conditions were applied in the $z$ direction to reduce the size effect and computational cost. During the cutting process using the nanostructured diamond tools, the influence of groove orientation, width, depth, factor and shape, which have a large influence on the subsurface and cutting force, could not be ignored. Therefore, the groove orientation $\left(0^{\circ}\right.$, $30^{\circ}, 60^{\circ}$ and $\left.90^{\circ}\right)$, width $(0.2,0.6,0.8$ and $1 \mathrm{~nm})$, depth $(0.2,0.6,0.8$

Table 1 Simulation parameters

Workpiece materials

Tool materials

Dimension of workpiece

Dimension of

structured diamond tool

Numbers of silicon

atoms in the workpiece

Depth of cut

Workpiece cutting surface

Initial temperature

Cutting distance

Time step

Cutting speed

Tool groove orientation

Tool groove depth

Tool groove width

Tool groove factor

Tool groove shape

\author{
Silicon \\ Diamond \\ $23 \mathrm{~nm} \times 8.2 \mathrm{~nm} \times 17.4 \mathrm{~nm}$ \\ $6 \mathrm{~nm} \times 5.5 \mathrm{~nm} \times 11.4 \mathrm{~nm}$
}

168864

$1 \mathrm{~nm}$

$\left[\begin{array}{lll}-1 & 0 & 0\end{array}\right]$ on $\left(\begin{array}{lll}0 & 1 & 0\end{array}\right)$ surface

$293 \mathrm{~K}$

0-18 nm

$1 \mathrm{fs}$

$200 \mathrm{~m} \mathrm{~s}^{-1}$

$0^{\circ}, 30^{\circ}, 60^{\circ}$ and $90^{\circ}$

$0.2,0.6,0.8$ and $1 \mathrm{~nm}$

$0.4,0.6$ and $0.8 \mathrm{~nm}$

$25,40,60$ and $85 \%$

Rectangular, arc, $\mathrm{V}$ and \#-shape and $1 \mathrm{~nm}$ ), factor $(25,40,60$ and $85 \%)$ and shape (rectangular, arc, $\mathrm{V}$ and \#-shape) were studied, as shown in Fig. 1(b). More structured tool parameters used in the present simulation are listed in Table 1, where groove factor is defined as the ratio of the nominal surface areas of structured and nonstructured tools. ${ }^{9}$

The accuracy of an MD simulation is governed by the interaction potential function, which, in turn, defines the reliability of simulation results. In this study, the Tersoff potential energy function was used in the simulation to describe interactions between workpiece atoms ( $\mathrm{Si}-\mathrm{Si}){ }^{\mathbf{1 5 , 2 0 - 2 6}}$ The Morse potential function was more appropriate for diatomic molecules; therefore, this study used the more physically significant Tersoff potential function to express interactions between workpiece atoms and tool atoms $(\mathrm{C}-\mathrm{Si}){ }^{24,25}$ Interactions between tool atoms $(\mathrm{C}-\mathrm{C})$ could not be ignored since the structured diamond tool was being modelled as a deformable body. Therefore, interactions between tool atoms (C-C) were also modelled using the Tersoff potential function. ${ }^{24-27}$

To reduce computational costs in this study, the cutting velocity used was $200 \mathrm{~m} \mathrm{~s}^{-1}$, which is much larger than the real velocity of cutting. However, based on previous work, ${ }^{15,23,26,28-34}$ MD simulations finished with a relatively high cutting speed can successfully produce key insights into the cutting process. All simulations were performed using open-source Large-scale Atomic/Molecular Massively Parallel Simulator (LAMMPS) software. ${ }^{35}$ OVITO software was used to visualize MD results. ${ }^{36}$

\section{Results and discussion}

\subsection{Effect of groove orientation}

Fig. 2 shows the bottom views of cutting tools with different groove orientations $\left(\theta: 0^{\circ}, 30^{\circ}, 60^{\circ}\right.$ and $\left.90^{\circ}\right)$. As shown in Fig. 2(a), $\theta$ is defined as the angle between the groove orientation and the $z$ axis. The corresponding simulations are shown in Fig. 3(a)-(d). During the cutting process, the workpiece atoms that moved on the top of the initial workpiece surface were regarded as chip atoms. When the location of a silicon atom was higher than the threshold height value $(h)$, the silicon atom was seen as a chip atom. In these simulations, the threshold height value was
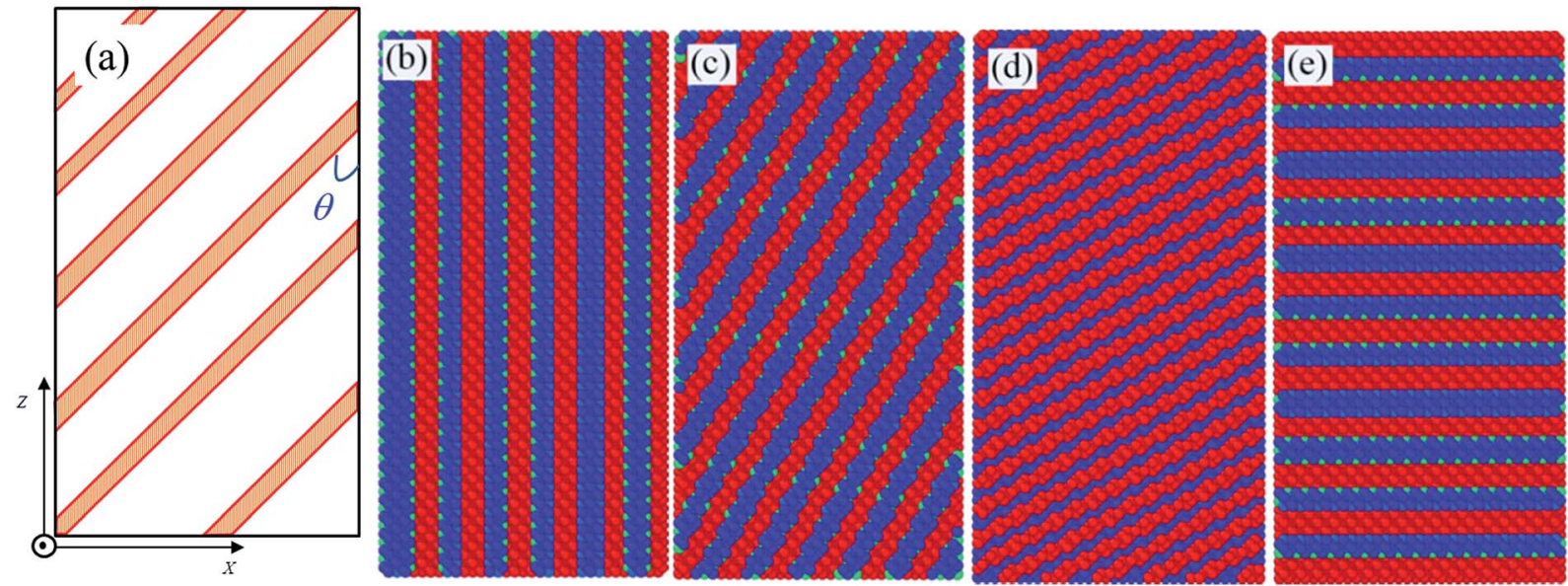

Fig. 2 (a) Schematic illustration of the cutting process with different groove orientations. Illustrations from studying the influence of groove orientations at (b) $0^{\circ}$, (c) $30^{\circ}$, (d) $60^{\circ}$ and (e) $90^{\circ}$. 
chosen as $0.27 \mathrm{~nm}$. Fig. 3(a)-(d) show top views of cutting surfaces for different groove orientations after the tool had advanced to $18 \mathrm{~nm}$. Fig. 3(f) shows the number of chip atoms related to cutting distance for different groove orientations. Fig. 3 shows that the chipping volume was largest at a $0^{\circ}$ groove orientation and smallest at a $90^{\circ}$ groove orientation. Furthermore, from Fig. 3(a)-(d), the volume of workpiece material pileup at both sides of the cutting groove was slightly affected by groove orientation. These results showed that cutting at a $0^{\circ}$ groove orientation produced the maximum material removal rate.

Von Mises stress assumes that the maximum deviatoric strain energy causes the material to yield, and is commonly used as the yield criterion to estimate material yielding. ${ }^{42}$ Von Mises stress also measures shear deformation, which governs shape changes, usually by activating defect transport mechanisms in continuous matter, whereas hydrostatic stress is associated with volume change leading to classic thermodynamic phase transitions. ${ }^{43}$ Therefore, hydrostatic stress and von Mises stress contribute to a material yielding, dislocation nucleation, motion and phase transitions. According to previous work, ${ }^{25,38}$ the stress tensor for an atom $(i)$ can be calculated using the following equation:

$$
S_{a b}=-\left[\begin{array}{l}
m v_{a} v_{b}+\frac{1}{2} \sum_{n=1}^{\mathrm{Np}}\left(r_{1 a} F_{1 b}+r_{2 a} F_{2 b}\right)+\frac{1}{2} \sum_{n=1}^{\mathrm{Nb}}\left(r_{1 a} F_{1 b}+r_{2 a} F_{2 b}\right) \\
+\frac{1}{3} \sum_{n=1}^{\mathrm{Na}}\left(r_{1 a} F_{1 b}+r_{2 a} F_{2 b}+r_{3 a} F_{3 b}\right) \\
+\frac{1}{4} \sum_{n=1}^{\mathrm{Nd}}\left(r_{1 a} F_{1 b}+r_{2 a} F_{2 b}+r_{3 a} F_{3 b}+r_{4 a} F_{4 b}\right) \\
+\frac{1}{4} \sum_{n=1}^{\mathrm{Ni}}\left(r_{1 a} F_{1 b}+r_{2 a} F_{2 b}+r_{3 a} F_{3 b}+r_{4 a} F_{4 b}\right) \\
+\sum_{n=1}^{\mathrm{Nf}} r_{i a} F_{i b}+\operatorname{Kspace}\left(r_{i a}, F_{i b}\right)
\end{array}\right]
$$
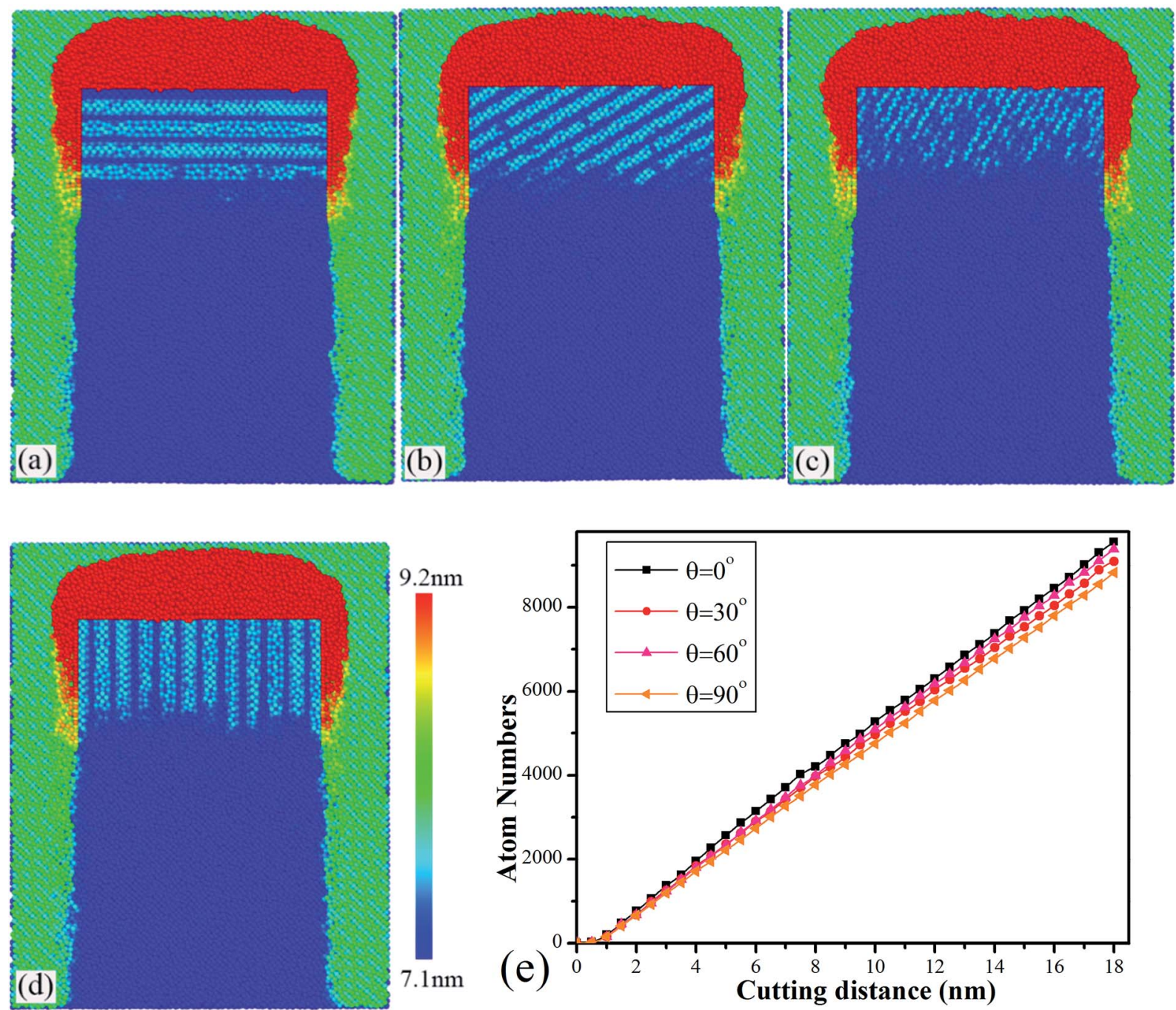

Fig. 3 Surface morphologies using different groove orientations of (a) $0^{\circ}$, (b) $30^{\circ}$, (c) $60^{\circ}$, and (d) $90^{\circ}$ after the tool has advanced by $18 \mathrm{~nm}$. Different atom heights are represented by different colors. (e) Number of atoms in chips vs. cutting distance. The groove depth, width, factor and shape are $0.6 \mathrm{~nm}, 0.6 \mathrm{~nm}, 40 \%$ and rectangular, respectively. 
where $a$ and $b$ take on values of $x, y, z$ to generate the six components of the symmetric tensor. The first term is the contribution of the kinetic energy of atom $i$. The second term is a pair-wise energy contribution, where $n$ loops over the $\mathrm{Np}$ neighbors of atom $i$, and $r_{1}$ and $r_{2}$ are the positions of the two atoms in the pair-wise interaction. $F_{1}$ and $F_{2}$ are the forces on the two atoms resulting from pairwise interactions. The third term is the bond contribution over the $\mathrm{Nb}$ bonds of atom $i$. Similarly, the $\mathrm{Na}$ angle, $\mathrm{Nd}$ dihedral, Ni improper interactions and Nf internal constraints of atom $i$ are accounted for in subsequent terms, while KSpace terms represent long-range coulombic interactions.

Von Mises and hydrostatic stresses are calculated using the following equations:

$$
\begin{aligned}
& \sigma_{\text {von Mises }}= \\
& \sqrt{\frac{\left(\sigma_{x x}-\sigma_{y y}\right)^{2}+\left(\sigma_{x x}-\sigma_{z z}\right)^{2}+\left(\sigma_{z z}-\sigma_{y y}\right)^{2}+6\left({\sigma_{x y}}^{2}+{\sigma_{y z}}^{2}+\sigma_{x z}{ }^{2}\right)}{2}}
\end{aligned}
$$

$$
\sigma_{\text {hydro }}=\frac{1}{3}\left(\sigma_{x x}+\sigma_{y y}+\sigma_{z z}\right)
$$

The local von Mises stress distribution and the hydrostatic stress distribution of a workpiece using a $30^{\circ}$ groove orientation cutting tool advancing to $18 \mathrm{~nm}$ are illustrated in Fig. 5(a) and (b), respectively. A similar trend was observed for other tool groove orientations and, therefore, was not repeated. Fig. 5(a) shows that high von Mises stress occurs in the contact zone between the workpiece and tool. However, Fig. 5(b) shows that a high-pressure phase transformation (HPPT) occurs in front of and beneath the tool edge. This HPPT promotes the transformation of silicon from brittle to ductile during contact loading. ${ }^{44}$ Phase transformation of brittle material silicon from Si-I (diamond cubic silicon) to Si-II (beta-silicon) is known to take place under pure hydrostatic stress of 11-12 Gpa. ${ }^{4,46}$ The various silicon phases during cutting of the ductile regime are summarized in Table 2. Therefore, the maximum value for hydrostatic pressure was $13 \mathrm{Gpa}$, which was high enough to initiate phase transformation in this study (Fig. 5(b)). Fig. 5(c) and (d) show variations in average von Mises stress and average hydrostatic stress of the subsurface with groove orientation, respectively. All von Mises and hydrostatic stresses were averaged over the cutting distance, from 6 to $18 \mathrm{~nm}$, cutting in a steady state. This clearly showed that both von Mises and

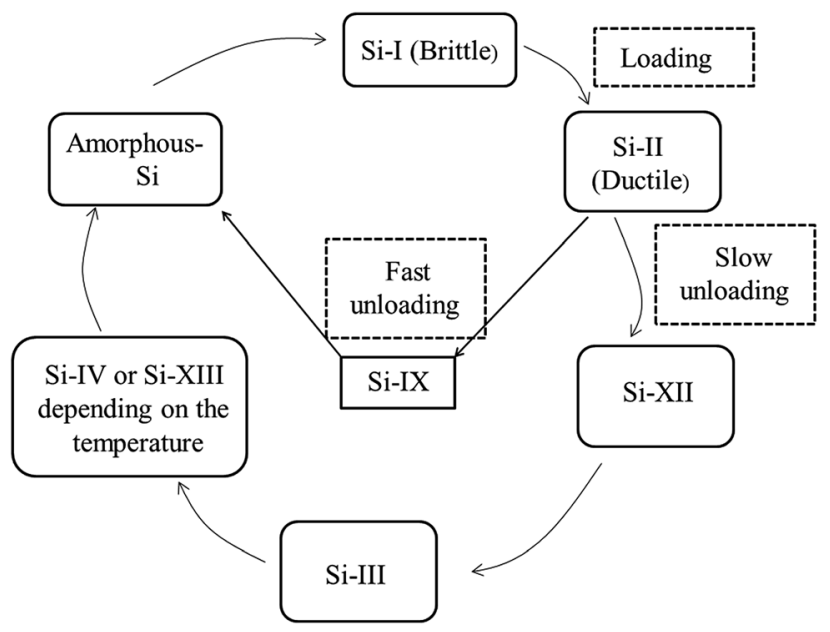

Fig. 4 High-pressure phase transformation of silicon during contact loading. ${ }^{37,38,41}$

hydrostatic stresses initially declined, and then increased as groove orientation increased, but produced two slightly different curves. Notably, both von Mises and hydrostatic stresses were smaller when cutting with a $60^{\circ}$ groove orientation tool compared with other structured tools, leading to smaller cutting forces, as shown in Fig. 8(b).

Fig. 6(a)-(d) show the coordination number (CN) of each silicon atom in the workpiece for different cutting tool groove orientations after the tool has advanced to $18 \mathrm{~nm}$. Silicon atoms exist in four-coordinate diamond cubic structures (Si-I) with a bond length $2.35 \AA$ at the initial cutting stage. As the diamond cutting tool advances, Si-II phase (beta-silicon, metallic and ductile) appears under a hydrostatic stress of 11-12 GPa. Subsequent increasing pressure produces other phases, such as Si-XI, Si-V, Si-VI, Si-VII and Si-X. ${ }^{45}$ Several phases of silicon are identified and summarized in Table 2, along with typical stress levels at which these phases persist. Reverse transformations from the Si-II phase depend on the rate of load release. Nonhydrostatic pressure could directly transform Si-I to a stable bct5 silicon phase. This cycle is shown in Fig. 4. Furthermore, the Si-II phase is six-coordinate body-centered tetragonal structure, including four neighbors at a distance of $2.42 \AA$ and two neighbors at $2.585 \AA$. The bct- $5 \mathrm{Si}$ crystalline structure contains one neighbor at $2.31 \AA$ and four neighbors at $2.44 \AA$. The Si-XII phase has four bonds of length $2.39 \AA$ and one of length $3.23 \AA$ or $3.36 \AA$, while Si-III has four bonds of length $2.37 \AA$ and one of length $3.41 \AA^{26,38}$ According to previous studies, ${ }^{26,34,38,45} 2.6 \AA$ is

Table 2 Various high-pressure phases of silicon $37-40$

\begin{tabular}{lll}
\hline Phase of silicon & Lattice structure & Stress (GPa) \\
\hline Pristine Si-I (brittle) & Diamond cubic & 0 to 12.5 \\
Si-II (metallic) & Beta-tin (body centered tetragonal) & 9 to 16 \\
Si-III or bc8 & BCC (body centered cubic) & 0 to 2.1 (ambient) \\
Si-XII (R8) & Rhombohedral & 2 to 12 \\
Si-IV & Hexagonal diamond (lonsdaleite) & Martensitic transformation from Si-I \\
Si-IX & St12, tetragonal & 0 to 12
\end{tabular}



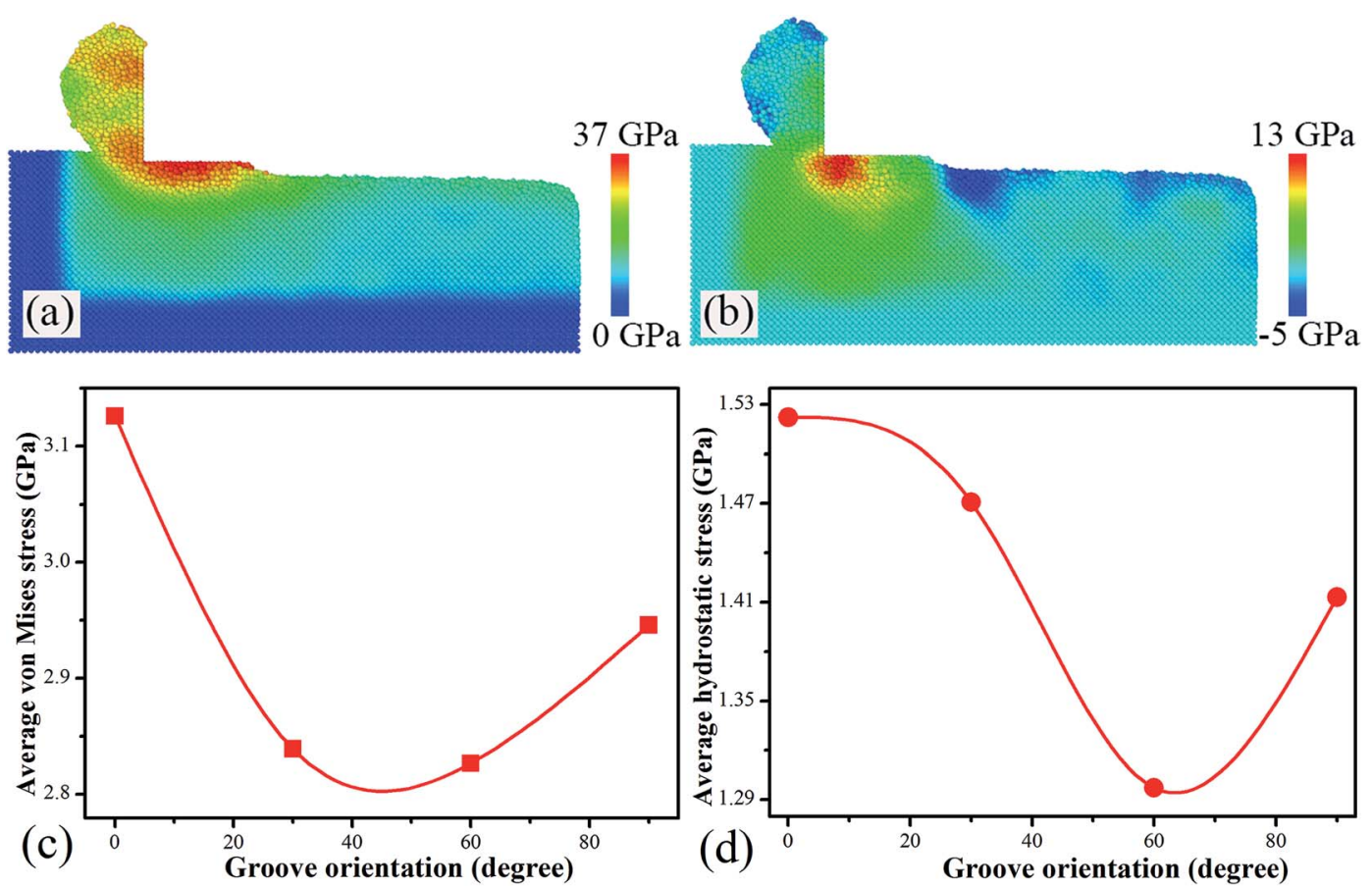

Fig. 5 (a) Von Mises stress distribution cutting for a tool travel distance of $18 \mathrm{~nm}$. (b) Hydrostatic stress distribution in machining at a cutting distance of $18.0 \mathrm{~nm}$. Groove orientation was $30^{\circ}$ for (a) and (b). (c) Average von Mises stress of subsurface at different groove orientations, and (d) average hydrostatic stress of subsurface at different groove orientations.

the cutoff radius for calculating the atomic coordination number of silicon atoms. Therefore, the five-coordinate atoms are bct5-Si phase, six-coordinate atoms are Si-II phase, and three-coordinate atoms are mainly surface atoms. ${ }^{34,47}$ Interestingly, Fig. 6(a)-(d) show that the distribution of crystalline phases in the transformation zone after cutting is consistent with the distribution of hydrostatic stress (as shown in Fig. 5(b)) because pressure is the main factor inducing phase transformation during the cutting process. ${ }^{38,47,48}$

Fig. 6(e)-(f) show variations in the number of Si-II and Bct5$\mathrm{Si}$ atoms with cutting distance for different groove orientations. Clearly, the number of Bct5-Si was much larger than that of Si-II during the nanoscale cutting process because of reverse transformation from Si-II. As shown in Fig. 4, upon load release after the cutting tool has passed the cutting area, Si-II transforms to atmospheric phase $\mathrm{Si}$ (a-Si), which causes a volume expansion of $\sim 10 \%$ and consequent elastic recovery of the cut surface. Additionally, Fig. 6(e)-(f) shows that, with increasing groove orientation $\theta$, the number of Si-II first increased and then abruptly decreased at $\theta=90^{\circ}$, but the number of bct5-Si remained similar. This means that more metallic and ductile Si-II phase formed from the original brittle Si-I phase with a cutting tool at a $60^{\circ}$ groove orientation.

Atoms of different lattice structures for different groove orientations after the tool advanced $18 \mathrm{~nm}$ are shown in Fig. 7(a)-(d), according to common neighbor analysis (CNA). ${ }^{49}$ Silicon atoms have been colored according to calculated CNA values, with blue representing Si-I phase, and gray representing other atoms, including Si-II, surface atoms and dislocation atoms. The groove orientation had a large influence on dislocation from the penetrated surface. Fig. 7(e) shows variations of other atoms with cutting distance for different cutting tool groove orientations. Other atoms clearly increased linearly as cutting distance increased in the four simulations. Fig. 7(e) shows that the number of other atoms at a cutting tool groove orientation of $0^{\circ}$ was slightly larger than those using other structured tools. This meant that, using a $0^{\circ}$ groove orientation cutting tool, more silicon atoms underwent phase transformation from Si-I phase (brittle) to other phases during the nanoscale cutting process.

To determine the effect of groove orientation on workpiece temperature, the average temperature evolution in the subsurface was plotted, as shown in Fig. 8(a). Cutting heat was generated by friction between the tool cutting edge and workpiece. The subsurface temperature rises rapidly during the initial cutting stage, and then reaches a steady state. Notably, a similar trend was observed for the subsurface cutting temperature using cutting tools with different groove orientations. However, with increasing groove orientation $(\theta)$, the subsurface temperature first decreased slightly and then abruptly increased at $\theta=90^{\circ}$, indicating that a groove orientation of $\theta=60^{\circ}$ was more conducive to reducing heat during the nanoscale cutting process. The relationships of tangential and normal cutting forces or frictional coefficients with the groove orientation at a cutting distance of $18 \mathrm{~nm}$ are shown in Fig. $8(\mathrm{~b})$ and (c). The lateral force (in the $z$ direction) is not shown because of fluctuations around an average value of zero during the scratching process due to balanced forces contributing from both sides of the groove. The friction coefficient is an important factor in the cutting process because it measures the ability to 

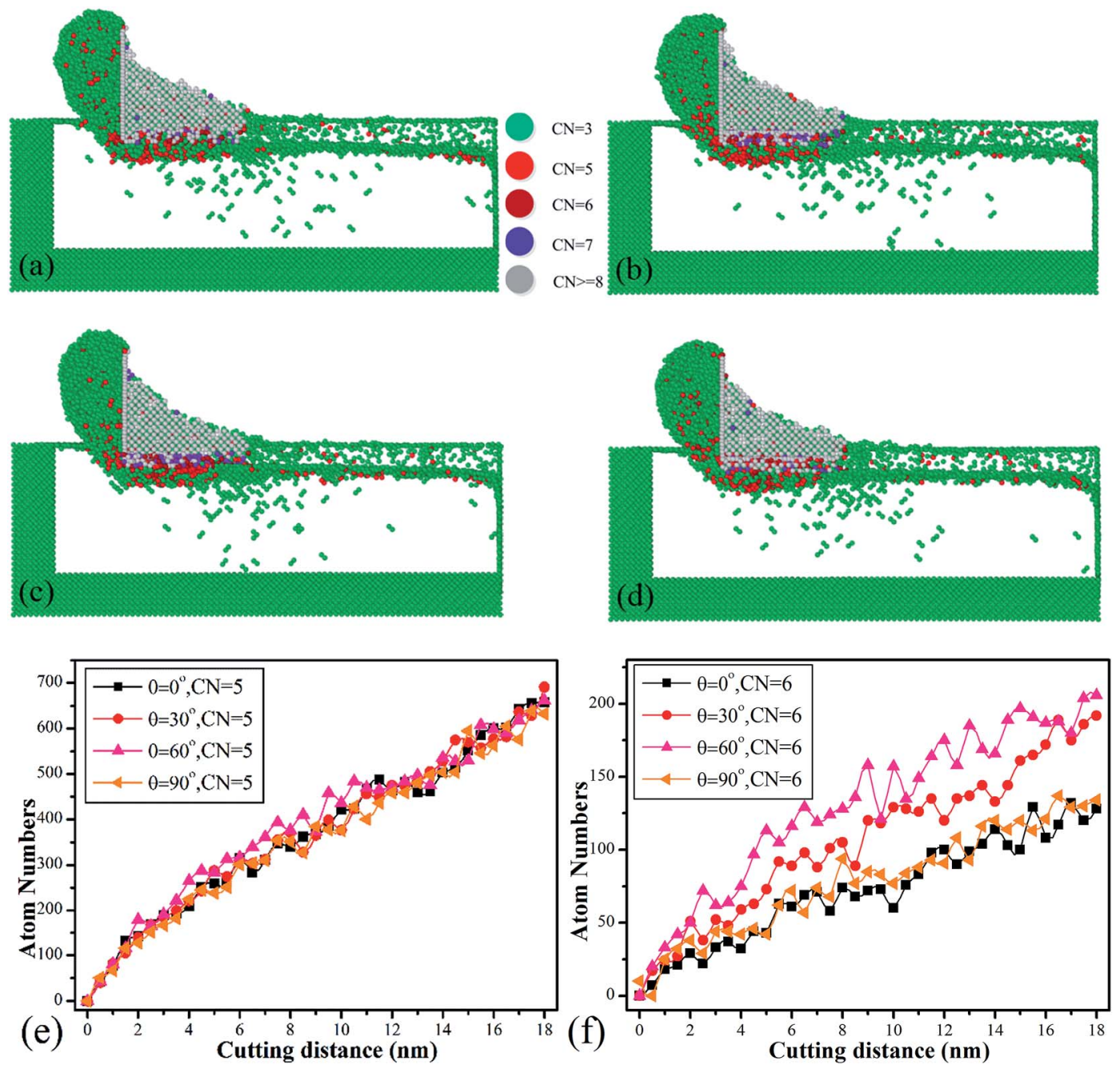

Fig. 6 Cross-sectional views of atomic positions for different groove orientations at an $18 \mathrm{~nm}$ cutting distance: (a) $0^{\circ}$, (b) $30^{\circ}$, (c) $60^{\circ}$, and (d) $90^{\circ}$. Coordination number (CN) of atoms are represented by different colors. (Four-coordinate diamond cubic silicon atoms are not shown.) (e and f) Number of atoms with specified number of nearest neighbors.

remove materials, and is defined as the tangential cutting force divided by the normal cutting force. Fig. 8(b) and (c) show that both tangential and normal cutting forces at a cutting tool groove orientation of $\theta=60^{\circ}$ are smaller than those using other structured tools, while the frictional coefficient is larger. Additionally, Fig. 8 (b) shows that groove orientation $(\theta)$ has little influence on the tangential force in cutting.

Fig. 9(a) shows the evolution of potential energy in subsurface atoms using different groove orientations. Evidently, as the tool advances, the potential energy increases rapidly. Nevertheless, the slope of potential energy against cutting distance was slightly larger during initial cutting than at the steady cutting stage. Moreover, comparison with tool cutting at a groove orientation of $90^{\circ}$ showed that a smaller groove orientation resulted in slightly higher potential energy in the subsurface, which indicated that the workpiece underwent different degrees of deformation during cutting processes using different cutting tool groove orientations. Fig. 9(b) shows the normal stresses $\left(\sigma_{x x}, \sigma_{y y}\right)$ and shear stress $\left(\tau_{x y}\right)$ in the subsurface during cutting as groove orientation was varied $\left(0^{\circ}, 30^{\circ}, 60^{\circ}\right.$ and $\left.90^{\circ}\right) . \sigma_{x x}$ and $\sigma_{y y}$ represent normal stresses in the $x$ and $y$ directions, while $\tau_{x y}$ represents shear stress in the $x$ direction. In Fig. 9(b), no obvious variation trends in $\sigma_{x x}, \sigma_{y y}$ and $\tau_{x y}$ were observed under different groove orientations, meaning that groove orientation had no obvious effect on stresses $\left(\sigma_{x x}, \sigma_{y y}\right.$ and $\tau_{x y}$ ). Furthermore, when $\theta=0^{\circ}$, normal stresses $\sigma_{x x}$ and $\sigma_{y y}$ were slightly more compressed, and shear stress $\tau_{x y}$ was slightly larger. Based on previous work, ${ }^{15,50,51}$ higher compressive stresses can accelerate the brittle-ductile transition of brittle silicon. A necessary condition for ductile cutting is that shear stress must be larger than material flow stress, so that dislocation emission takes place. Therefore, this result means that tools with a groove orientation of $0^{\circ}$ tend to cut silicon in a more ductile mode than other structured tools.

\subsection{Effect of groove depth}

Fig. 10 shows cutting tool models with different groove depths. Fig. 10(a)-(d) represent tools with groove depths of 0.2, 0.6, 0.8 

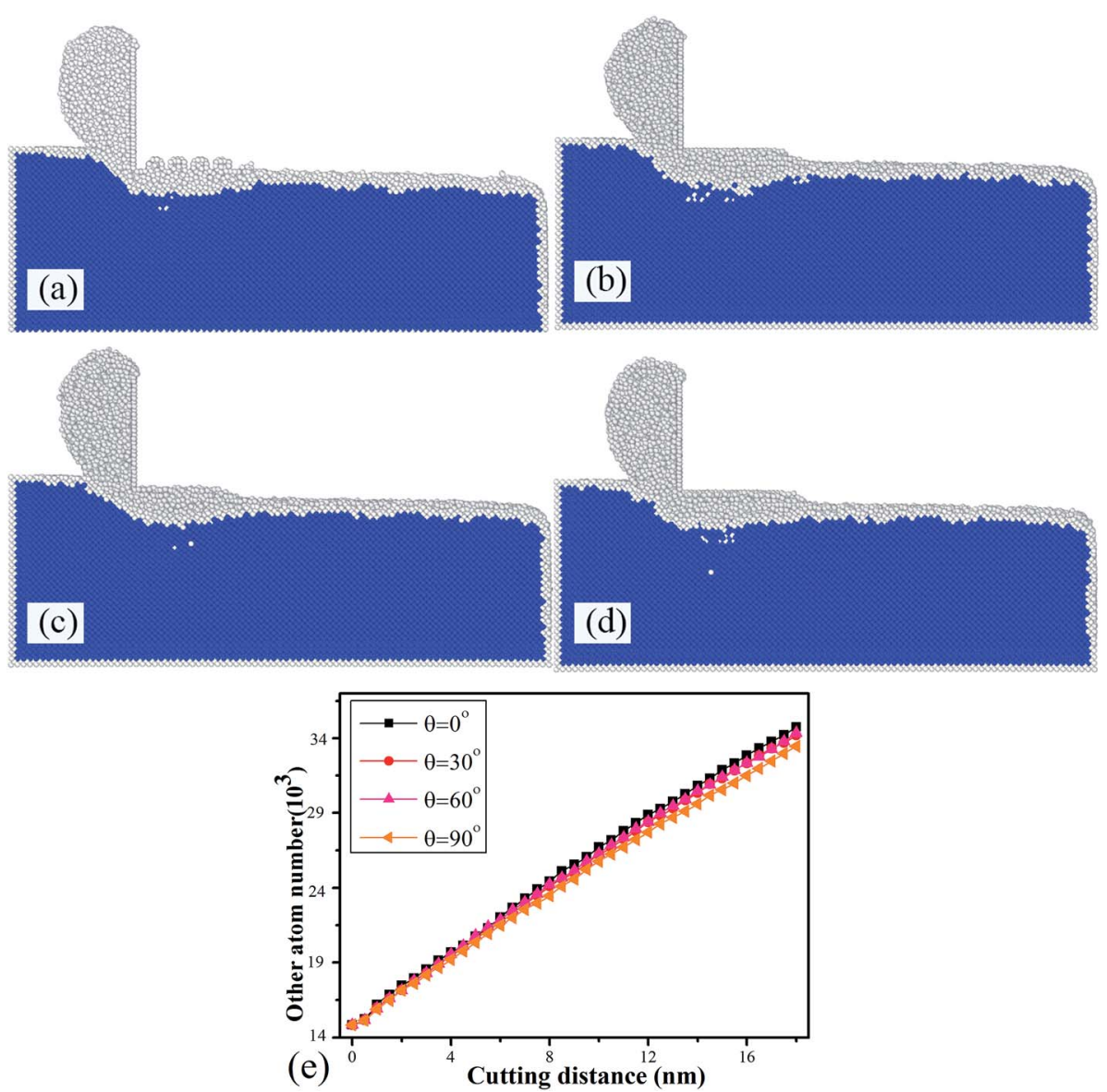

Fig. 7 Cross-sectional views of different groove orientations: (a) $0^{\circ}$, (b) $30^{\circ}$, (c) $60^{\circ}$, and (d) $90^{\circ}$ at a cutting distance of $18 \mathrm{~nm}$. Atoms are colored according to calculated CNA values; diamond cubic crystal structure in blue, other atoms in gray. (e) Evolution of other atoms at different groove orientations.

and $1 \mathrm{~nm}$, respectively. Corresponding $\mathrm{MD}$ simulations are shown in Fig. 11(a)-(d). Fig. 11(a)-(d) show the machined surface morphologies using different groove depths after a tool advance of $18 \mathrm{~nm}$. The volume of workpiece material pileup on both sides of the groove was strongly influenced by groove depth in nanoscale cutting. This means that the quality of ground surface was strongly affected by groove depth. Fig. 4(f) shows variations in the number of chip atoms with cutting distance using different groove depths. Although the curve trends were nearly the same, a groove depth of $0.2 \mathrm{~nm}$ resulted in more chips than other structured tool cutting, and the number of chip atoms with a groove depth of $1 \mathrm{~nm}$ was slightly smaller. These results indicated that a smaller groove depth $(0.2$ $\mathrm{nm}$ ) cutting tool produced a slightly stronger material removal ability in nanoscale cutting.

The von Mises stress distribution for workpiece atoms using cutting tools of different groove depths after $18 \mathrm{~nm}$ of tool advance is shown in Fig. 12(a)-(d). The highest von Mises stress existed in the chip and at the workpiece-tool interface. The average von Mises stresses of the subsurface at different groove depths are plotted in Fig. 12(e). With increasing groove depth, von Mises stress first declines rapidly, and then rises even faster. Moreover, by comparing tool cutting with four different groove depths, the average von Mises stress produced by a groove depth of $0.8 \mathrm{~nm}$ was smaller than those of other structured tools, resulting in smaller shear deformation, which governs shape changes, usually by activating defect transport mechanisms. ${ }^{43}$

Fig. 13(a) shows the average variation in subsurface temperature over the cutting distance. With increasing groove depth, the average temperature fluctuated slightly in a small range during the steady cutting stage. This meant that groove depth had little influence on subsurface temperature. The average tangential force in the $x$ direction and average normal force in the $y$ direction for different groove depths during cutting are calculated in Fig. 13(b). The comparison of cutting forces clearly shows that the normal cutting force decreased as the groove depth increased, while the tangential force initially decreased, and then increased. Furthermore, cutting with a groove depth of $0.8 \mathrm{~nm}$ produced a tangential force larger than that using a groove depth of $0.6 \mathrm{~nm}$, while the resultant cutting force was the smallest. This result means that a cutting tool with a groove depth of $0.8 \mathrm{~nm}$ can reduce the cutting force during the nanoscale cutting process. The average normal stresses $\left(\sigma_{x x}, \sigma_{y y}\right)$ and shear stress $\left(\tau_{x y}\right)$ in the subsurface were also calculated, as shown in Fig. 13(c). As the groove depth increased, the shear 

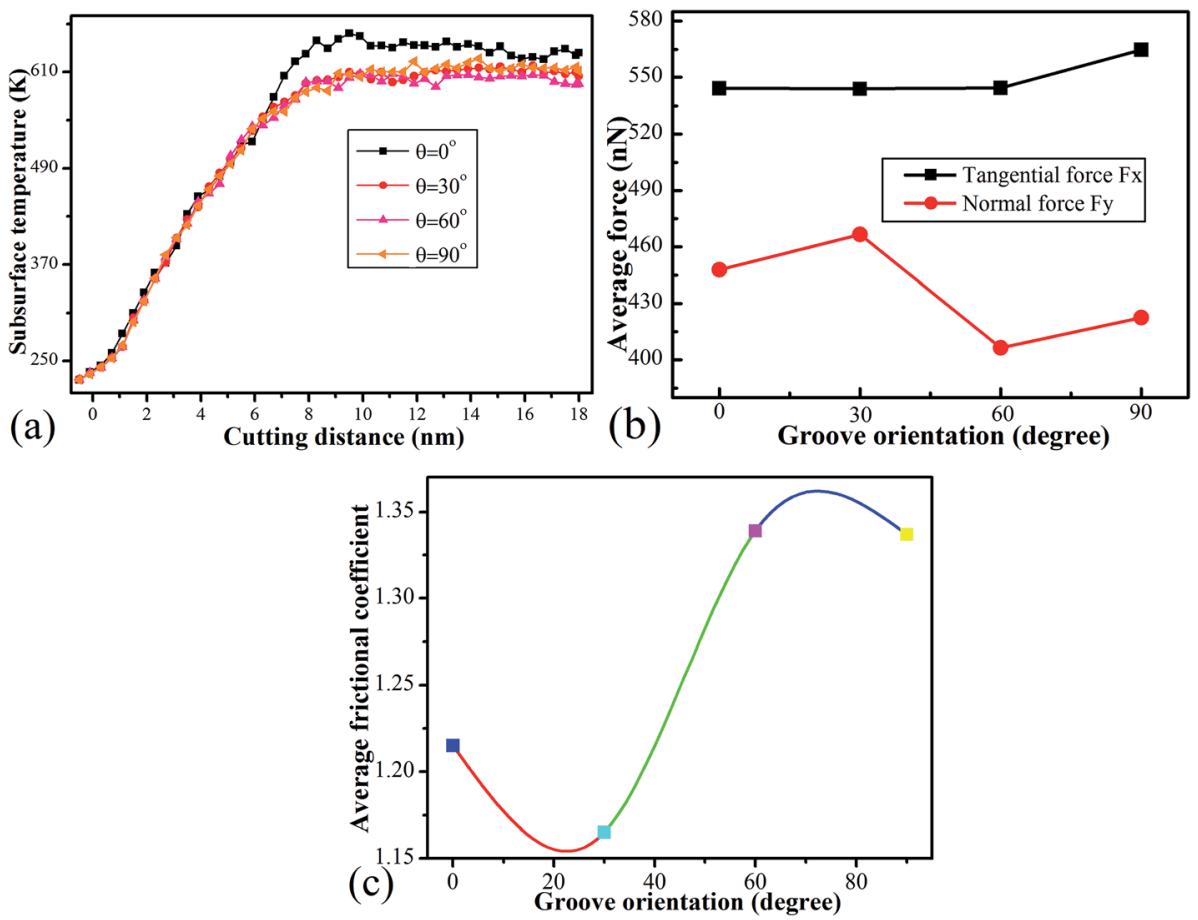

Fig. 8 (a) Subsurface temperature variations in the workpiece, (b) variation in the average cutting force and (c) frictional coefficients at different groove orientations.
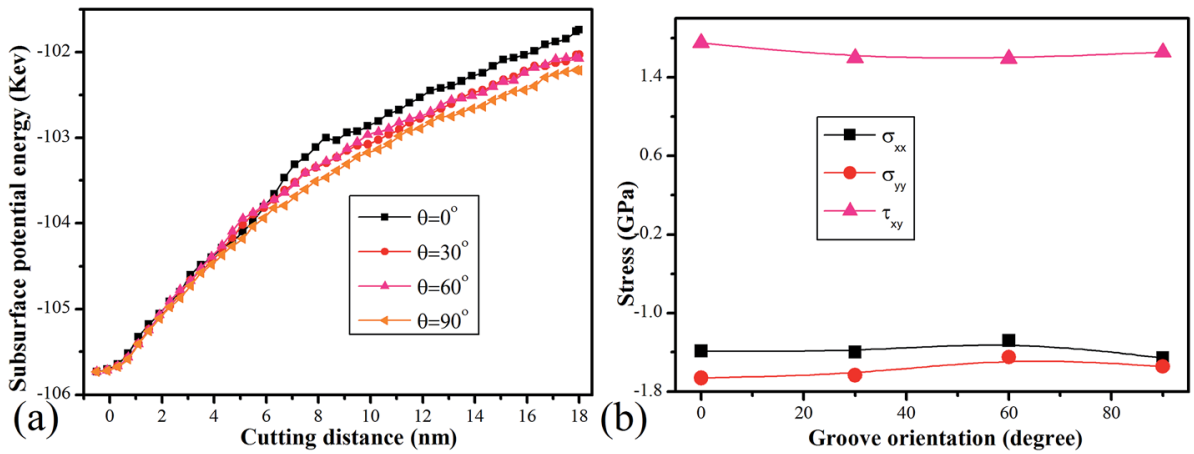

Fig. 9 (a) Evolution of potential energy in the workpiece subsurface. (b) Normal stresses $\left(\sigma_{x x}, \sigma_{y y}\right)$ and shear stress $\left(\tau_{x y}\right)$ in the subsurface during cutting with nanostructured tools of different groove orientations.

stress $\left(\tau_{x y}\right)$ remained almost the same, but the normal stresses $\left(\sigma_{x x}\right.$ and $\left.\sigma_{y y}\right)$ initially increased slightly, and then decreased slightly. Furthermore, the curve trends for $\sigma_{x x}$ and $\sigma_{y y}$ were almost identical. In addition, the compressive stresses $\left(\sigma_{x x}\right.$ and $\left.\sigma_{y y}\right)$ and shear stress $\left(\tau_{x y}\right)$ were observed to be largest when using a groove depth of $0.2 \mathrm{~nm}$. This result showed that tools with a groove depth of $0.2 \mathrm{~nm}$ tend to cut silicon in a more ductile mode than other structured tools.

\subsection{Effect of groove width}

The surface morphologies of diamond tools with different groove widths are shown in Fig. 14. Fig. 14(a)-(a) represent tools

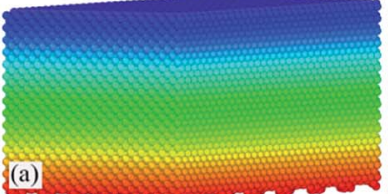

(a)

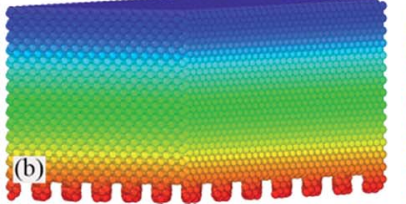

\section{(c)} (c)

Fig. 10 Cutting tools with different groove depths: (a) $0.2 \mathrm{~nm}$, (b) $0.6 \mathrm{~nm}$, (c) $0.8 \mathrm{~nm}$, and (d) $1 \mathrm{~nm}$. 

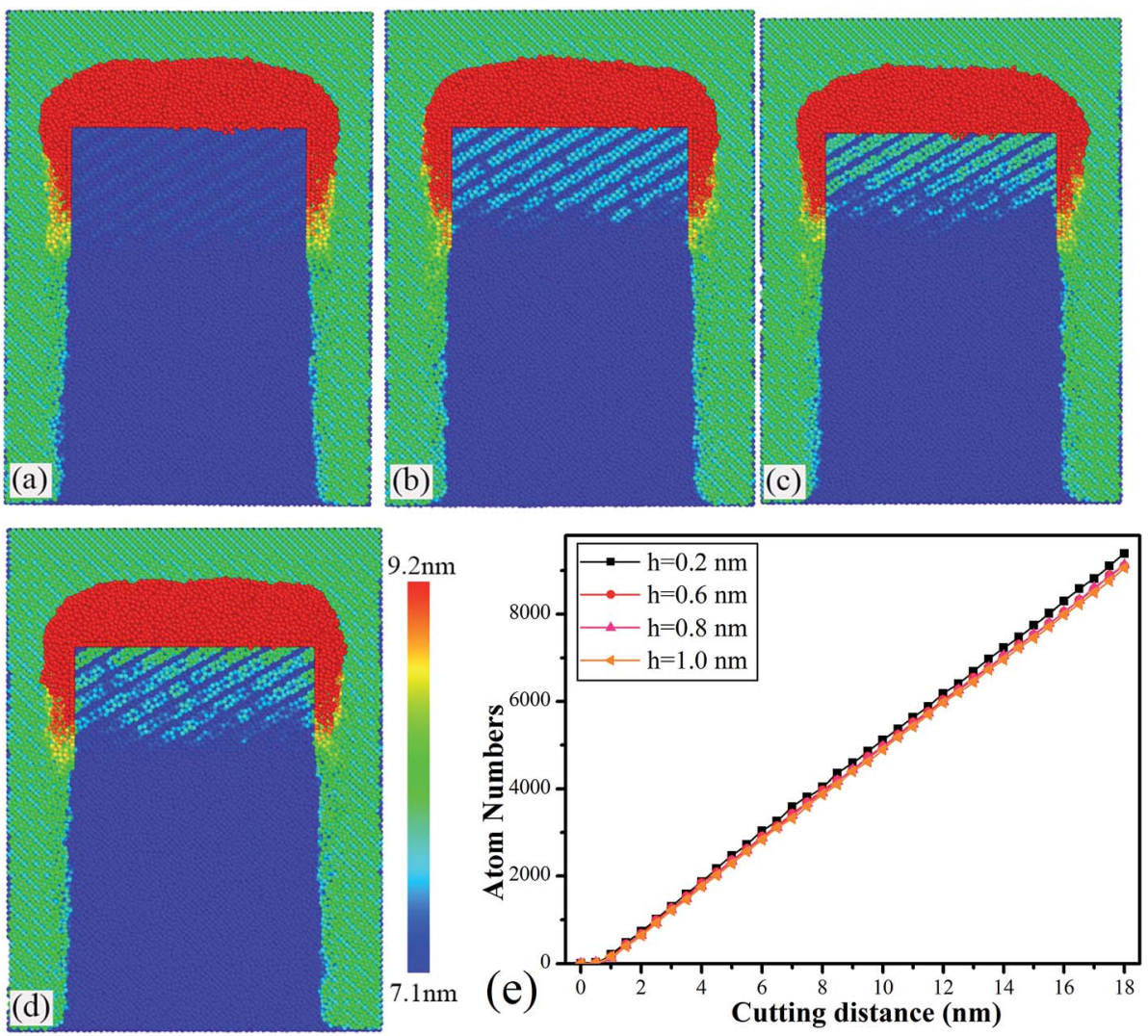

Fig. 11 Cross-sectional snapshots of workpiece during nanometric cutting process at groove depths of (a) $0.2 \mathrm{~nm}$, (b) $0.6 \mathrm{~nm}$, (c) $0.8 \mathrm{~nm}$, and (d) $1 \mathrm{~nm}$. (e) Number of atoms in chips during nanoscale cutting of silicon in four cases. Atoms are colored according to atomic height. The groove orientation, width, factor and shape are $30^{\circ}, 0.6 \mathrm{~nm}, 40 \%$ and rectangular, respectively.

with groove widths of $0.4 \mathrm{~nm}, 0.6 \mathrm{~nm}$ and $0.8 \mathrm{~nm}$, respectively, and the corresponding simulations are illustrated in Fig. 15(a)(c), which show chips of the deformed single crystal silicon at a cutting distance of $18 \mathrm{~nm}$ using different groove widths. Fig. 15(d) shows chip atom displacement during the cutting process. With increasing groove depth, the number of chip atoms decreased. This result indicated that cutting with a smaller groove width gave a larger material removal rate than large groove width. Furthermore, from Fig. 15(a)-(c), the volume of workpiece material pileup was smallest when cutting with a groove width of $0.4 \mathrm{~nm}$, meaning that a groove width of $0.4 \mathrm{~nm}$ obtained the best ground surface quality.

A comparison of the evolution of surface cutting temperature over a cutting distance of $18 \mathrm{~nm}$ in all the three cases is shown in Fig. 16(a). Average subsurface temperature decreased as groove width increased, indicating that a cutting tool with a smaller groove width resulted in more cutting heat during the nanoscale cutting process. Correspondingly, the variation in tangential forces $\left(F_{x}\right)$ and normal forces $\left(F_{y}\right)$ with cutting distance are also plotted in Fig. 16(b). With increasing groove width, the normal forces $\left(F_{y}\right)$ clearly decreased, while the tangential forces $\left(F_{x}\right)$ initially decreased, and then slightly increased. In general, an increase in groove width caused a decrease in resultant cutting force. Variations in average normal stresses $\left(\sigma_{x x}, \sigma_{y y}\right)$ and shear stress $\left(\tau_{x y}\right)$ in the subsurface with groove width are shown in Fig. 16(c). Clearly, increasing groove width caused a slight increase in average normal stresses ( $\sigma_{x x}$ and $\sigma_{y y}$ ), but the shear stress $\left(\tau_{x y}\right)$ slightly declined initially, and then increased slightly. Furthermore, using a cutting tool groove width of $0.4 \mathrm{~nm}$, the compressive stresses (including $\sigma_{x x}$ and $\left.\sigma_{y y}\right)$ and shear stress $\left(\tau_{x y}\right)$ were slightly larger than those using a larger groove width tool, as shown in Fig. 16(c). This result showed that tools with a groove width of $0.4 \mathrm{~nm}$ tended to cut silicon in a more ductile mode than other structured tools.

\subsection{Effect of groove factor}

The surface morphologies of structured diamond tools with different groove factors, and the corresponding workpiece after the tool has advanced by $18 \mathrm{~nm}$, are shown in Fig. 17 and Fig. 18(a)-(d), respectively. Variations in chip atoms during the nanoscale cutting process are plotted in Fig. 18(e) for cutting with different groove factors. Although a similar trend was observed for all four simulations, the number of chip atoms slightly increased as the groove factor increased, as shown in Fig. 18(e). This showed that a larger groove factor tool resulted in a slightly stronger material removal ability during nanoscale cutting. Furthermore, a comparison of groove factors of $25 \%$ and $85 \%$ showed that the volume of material pileup on both sides of the cutting groove varied only slightly during cutting. This means that the quality of the ground surface was nearly independent of the groove factor. 

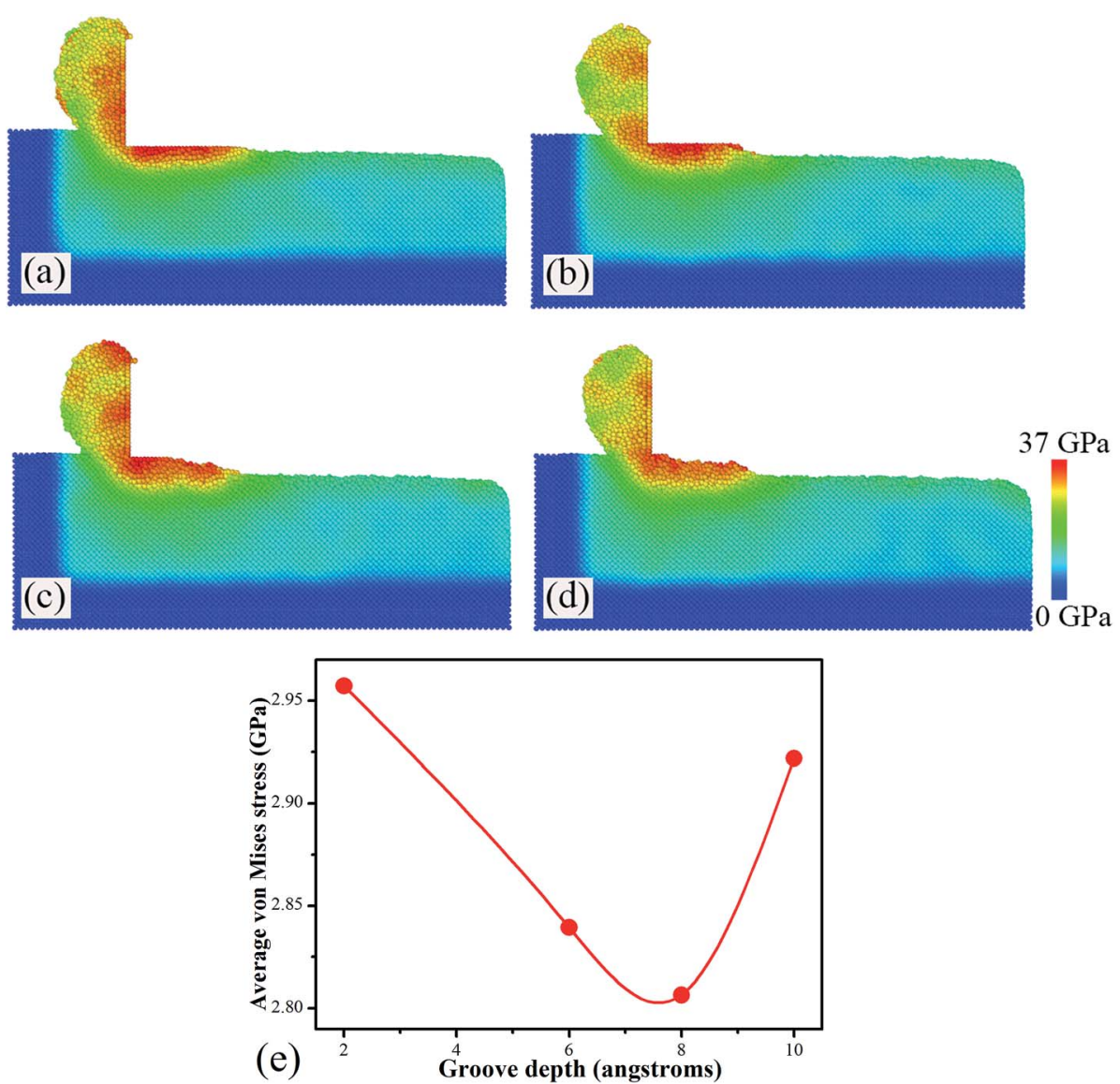

Fig. 12 Von Mises stress distribution at a cutting distance of $18 \mathrm{~nm}$ using different groove depths: (a) $0.2 \mathrm{~nm}$, (b) $0.6 \mathrm{~nm}$, (c) $0.8 \mathrm{~nm}$, and (d) $1 \mathrm{~nm}$. (e) Average von Mises stress of subsurface at different groove depths.
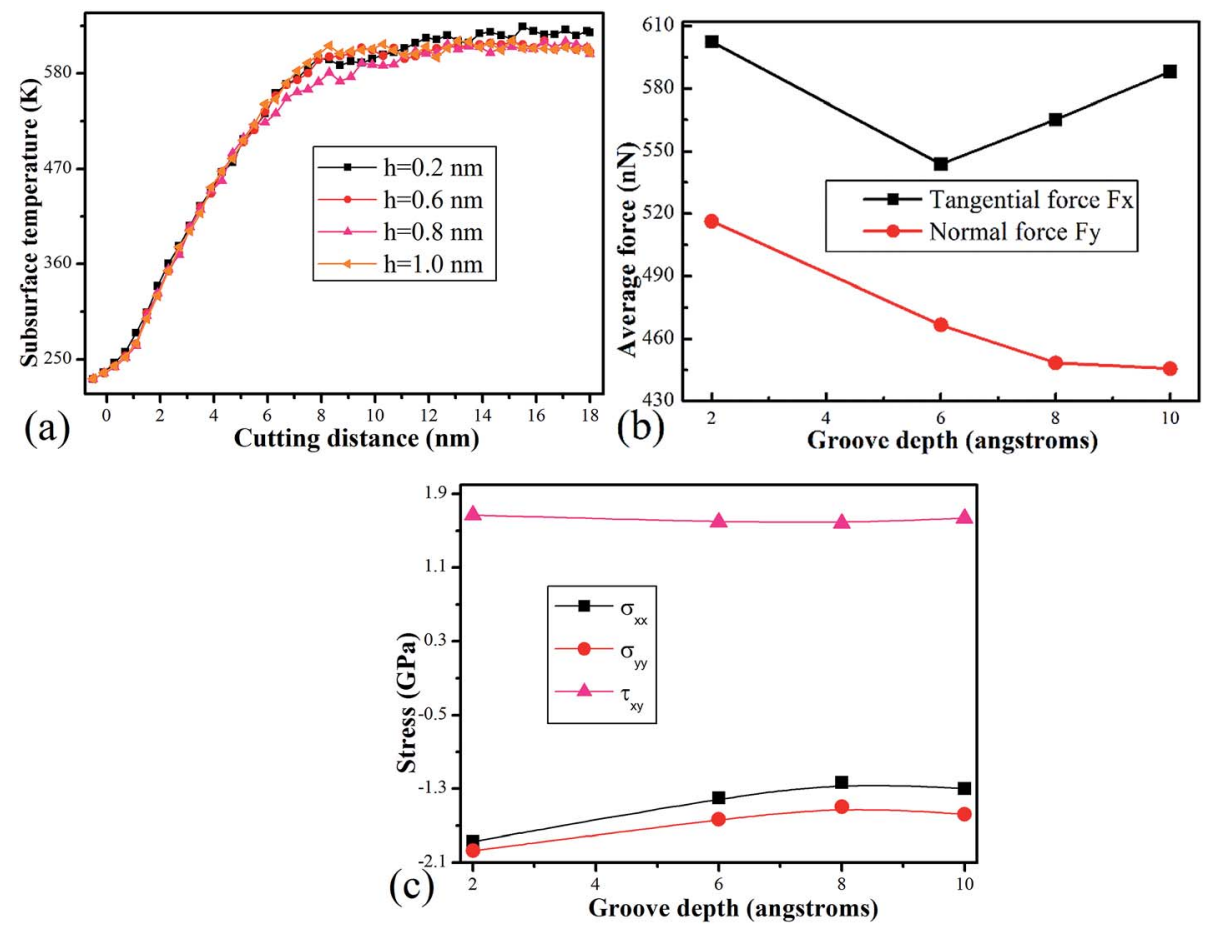

Fig. 13 (a) Variation in average subsurface temperature, (b) average cutting force, and (c) average normal stresses $\left(\sigma_{x x}, \sigma_{y y}\right)$ and shear stress $\left(\tau_{x y}\right)$ in the subsurface using different groove depths. 

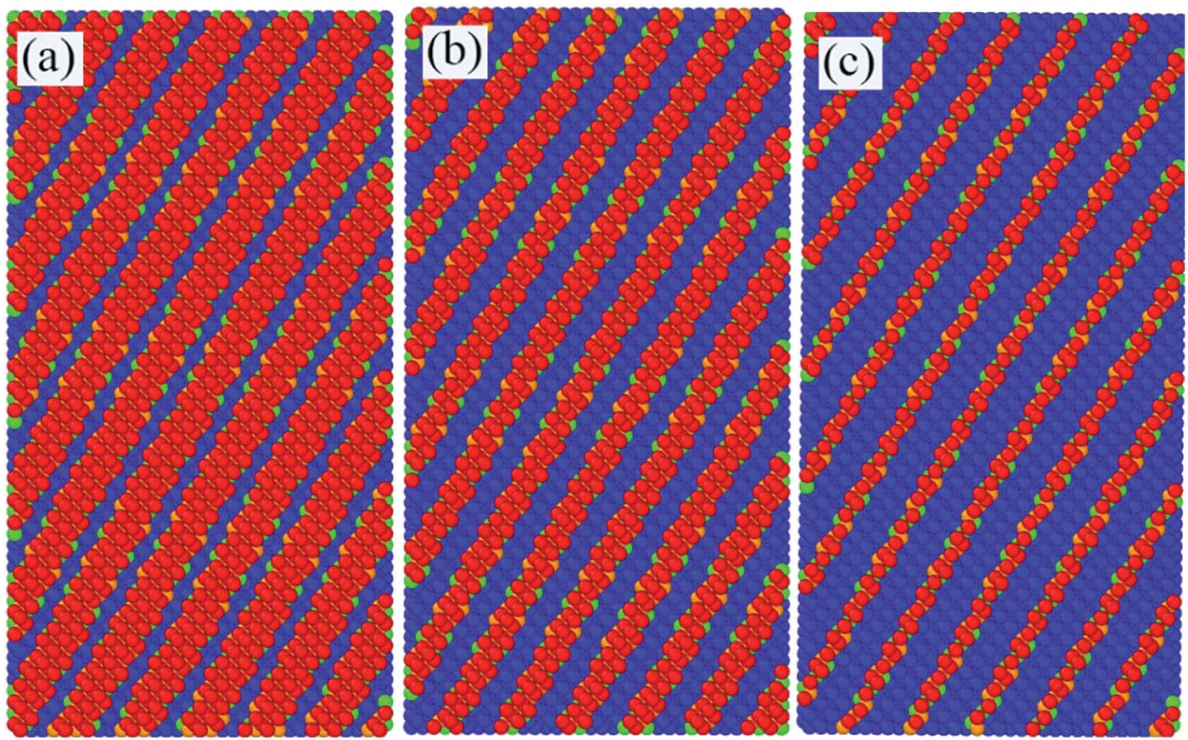

Fig. 14 Surface morphologies of cutting tools with different groove widths: (a) $0.4 \mathrm{~nm}$, (b) $0.6 \mathrm{~nm}$, and (c) $0.8 \mathrm{~nm}$.
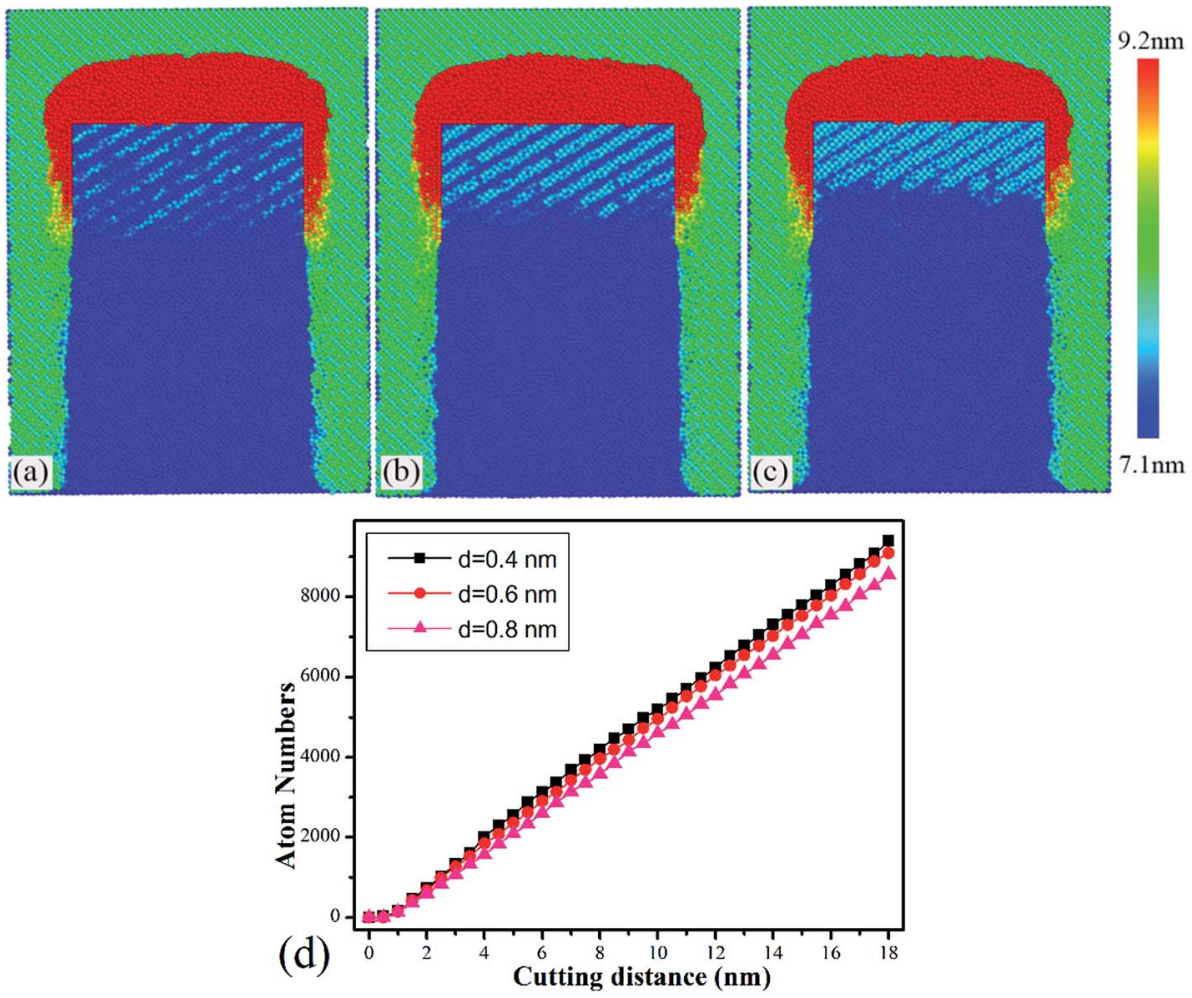

Fig. 15 Top view of cutting surface using different groove widths: (a) $0.4 \mathrm{~nm}$, (b) $0.6 \mathrm{~nm}$, and (c) $0.8 \mathrm{~nm}$. (d) Number of atoms in chips during nanoscale cutting of silicon in three cases. Atoms are colored according to their height. The groove orientation, depth, factor and shape are $30^{\circ}$, $0.6 \mathrm{~nm}, 40 \%$ and rectangular, respectively.

Fig. 19(a) shows the average rise in subsurface temperature during the cutting process. Increasing the groove factor, $\eta$, caused the average subsurface temperature to first increase, and then abruptly decrease slightly, at $\eta=85 \%$. This may be caused by the groove accelerating heat dissipation in the subsurface atoms. Fig. 19(b) shows the average force components for different groove factors. It was found that the tangential force changed slightly with the increasing groove factor, but the normal force changed significantly. Furthermore, a comparison of different groove factors indicated that a groove factor of $\eta=$ $40 \%$ reduced the overall magnitude of resultant cutting forces. Fig. 19(c) shows variations in normal stresses $\left(\sigma_{x x}\right.$ and $\left.\sigma_{y y}\right)$ and 

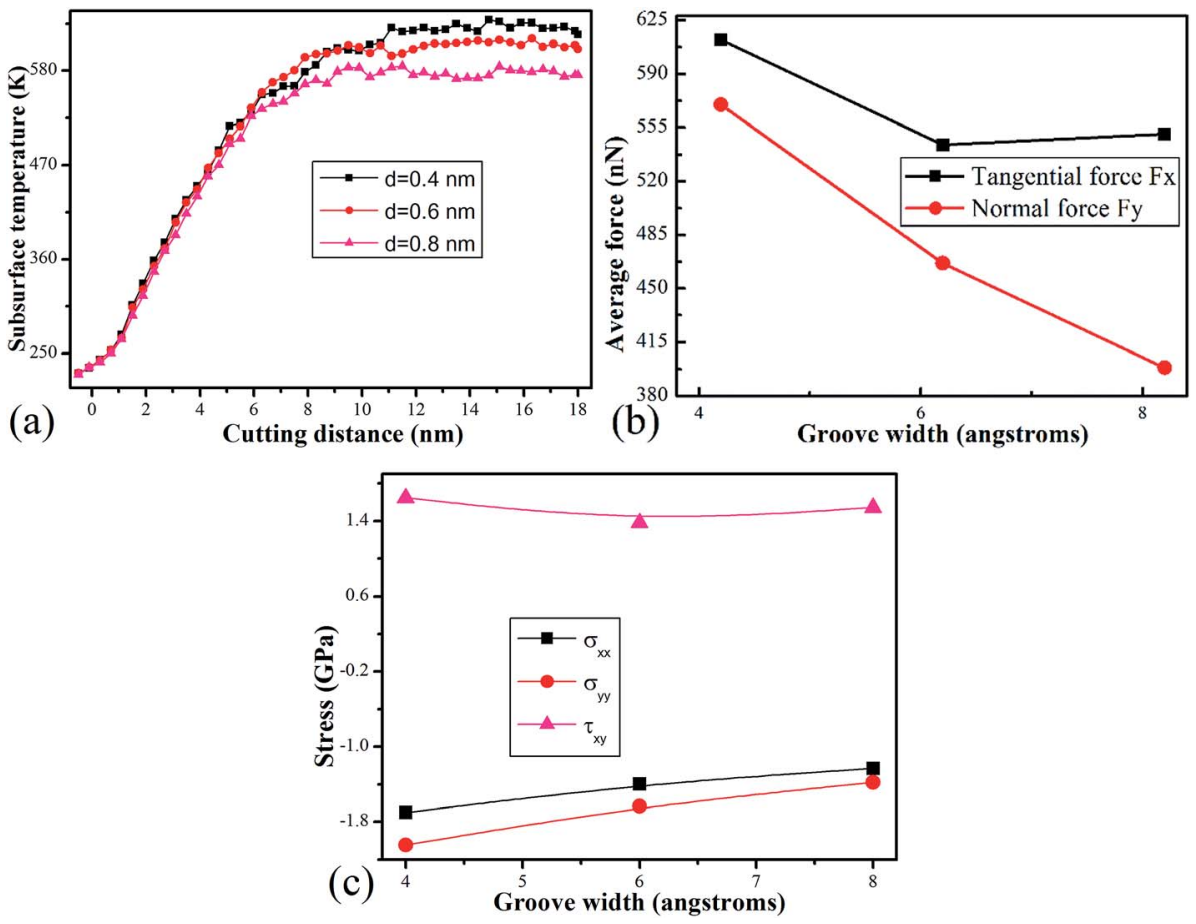

Fig. 16 Effect of groove width on (a) average subsurface temperature, (b) cutting force, and (c) average normal stresses ( $\left.\sigma_{x x}, \sigma_{y y}\right)$ and shear stress $\left(\tau_{x y}\right)$ in the subsurface.
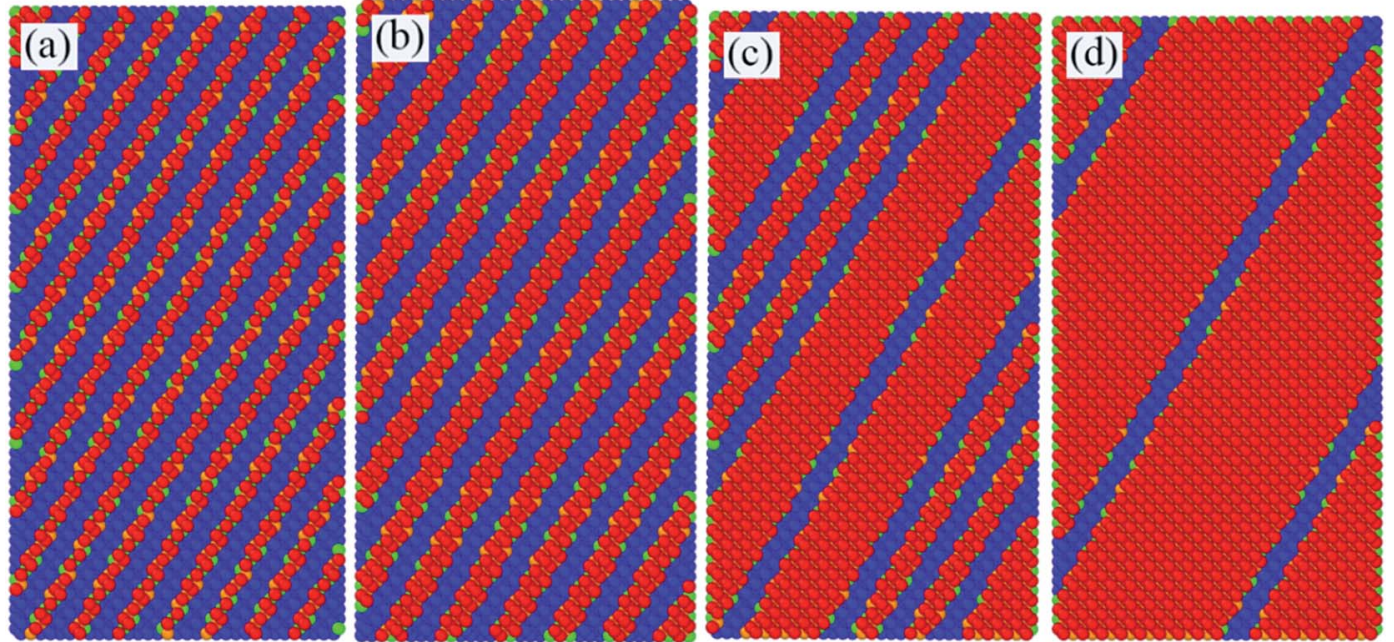

Fig. 17 Surface morphologies of cutting tools with different groove factors: (a) $25 \%$, (b) $40 \%$, (c) $60 \%$, and (d) $85 \%$.

shear stress $\tau_{x y}$ with changing groove factor, showing that compressive stresses $\left(\sigma_{x x}\right.$ and $\left.\sigma_{y y}\right)$ increase with the increasing groove factor, while shear stress $\left(\tau_{x y}\right)$ also increases slightly. The curve trends for $\sigma_{x x}$ and $\sigma_{y y}$ were slightly different, indicating that a larger cutting tool groove factor might lead to a more ductile cutting mode than a smaller groove factor.

\subsection{Effect of groove shape}

Diamond tools with different groove shapes and the corresponding spatial distributions of workpiece atoms with a cutting distance of $18 \mathrm{~nm}$ are shown in Fig. 20(a)-(h), and the number of chip atoms against displacement are shown in Fig. 20(i). The number of chip atoms rapidly increased at an approximately constant rate with increasing cutting distance. This was due to motion of the tool causing more and more workpiece atoms to accumulate and pile up in front, and on both sides, of the tool. A comparison of different tool groove shapes showed that when the structured tool had a V-shaped groove, the material removal rate was the largest. However, if the structured tool had a rectangular groove, the material 

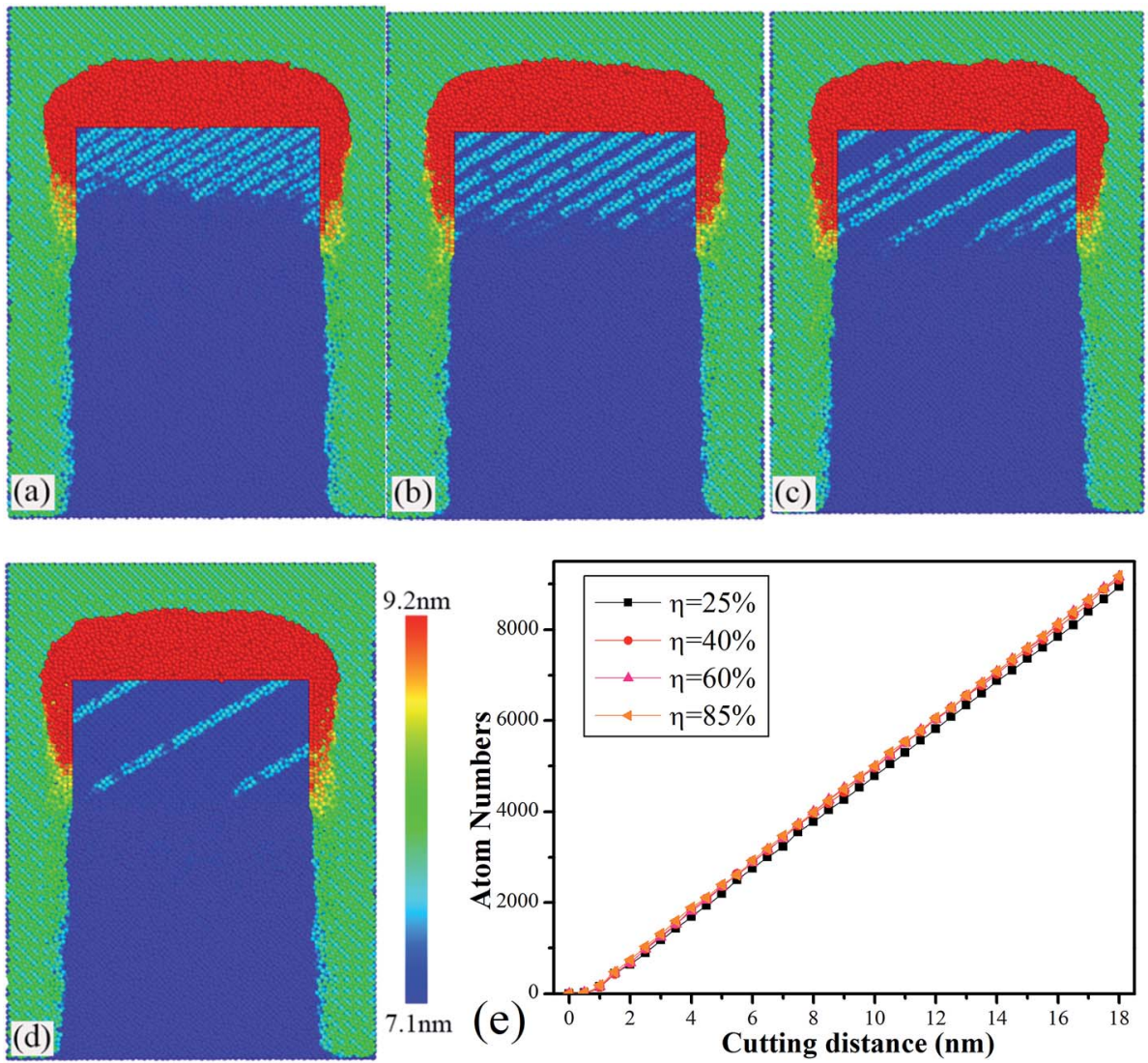

Fig. 18 Influence of groove factors of (a) $25 \%$, (b) $40 \%$, (c) $60 \%$, and (d) $85 \%$. (e) Number of atoms in chips during nanoscale cutting of silicon in four cases. Atoms are colored according to height. The groove orientation, depth, width and shape are $30^{\circ}, 0.4 \mathrm{~nm}, 0.6 \mathrm{~nm}$ and rectangular, respectively.
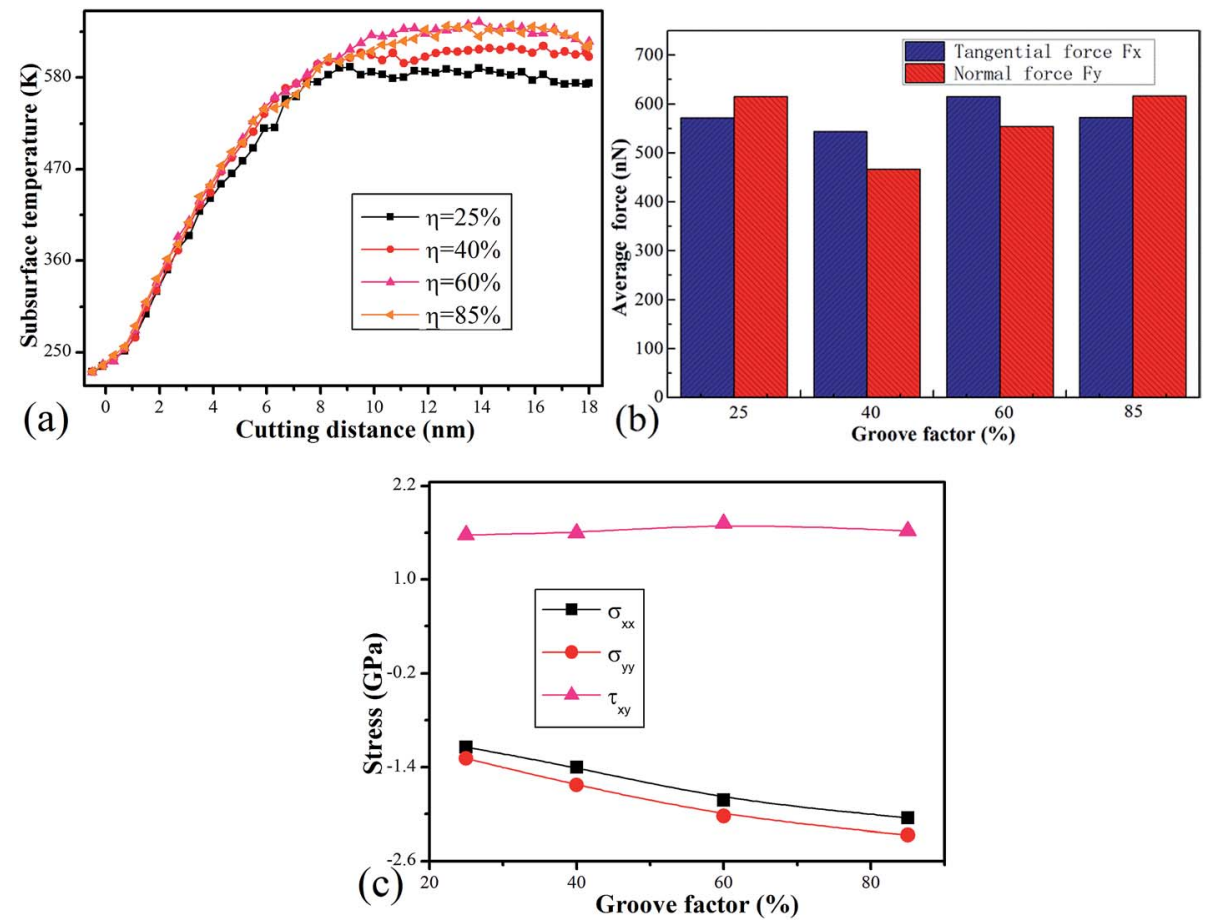

Fig. 19 Variations in (a) subsurface temperature, (b) average cutting forces, and (c) normal stresses $\left(\sigma_{x x}, \sigma_{y y}\right)$ and shear stress $\left(\tau_{x y}\right)$ using different groove factors. 

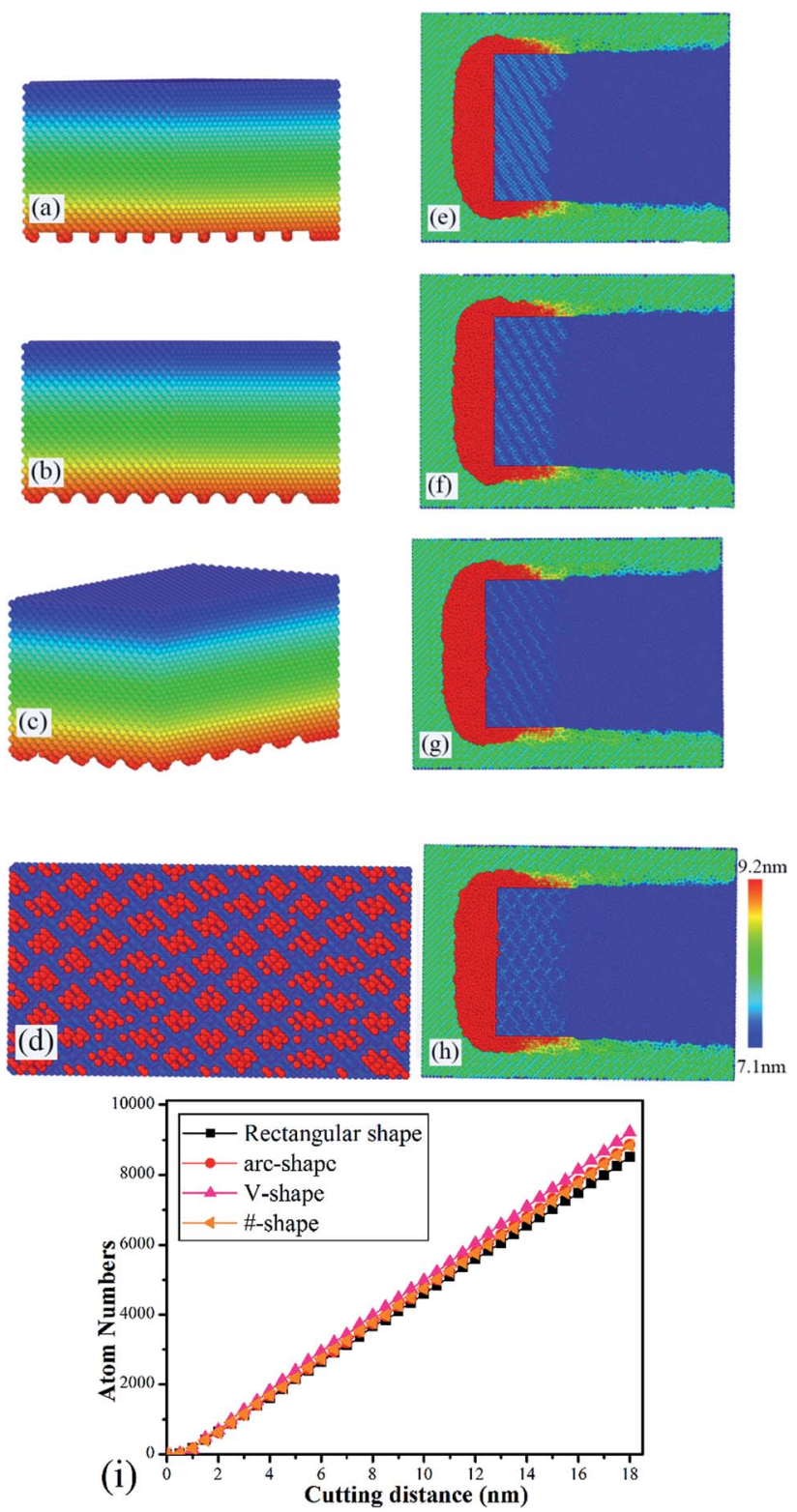

Fig. 20 Workpiece surface morphology after cutting using different groove shapes: ( $a$ and e) rectangular groove, (b and f) arc groove, (c and g) $\mathrm{V}$-shape groove, and ( $d$ and h) \#-shape groove. (e) Number of atoms in chips during nanoscale cutting of silicon in four cases. Atoms are colored according to their height. The groove orientation, depth, width and factor are $30^{\circ}, 0.4 \mathrm{~nm}, 0.8 \mathrm{~nm}$ and $27.27 \%$, respectively.

removal rate was the smallest. This showed that V-shape groove cutting can improve the material removal ability in nanoscale cutting. Furthermore, different groove shapes cutting generated almost identical volumes of workpiece material pileup on both sides of the cutting groove, as shown in Fig. 20(e)-(h), meaning that groove shape had little effect on ground surface quality during the nanoscale cutting process.

\section{Conclusions}

Large-scale MD simulations using the Tersoff potential have been carried out to study the effects of different groove directions, depths, widths, factors and shapes on the nanoscale cutting process. Accordingly, the following conclusions can be drawn:

(1) Groove direction has a significant effect on the nanoscale cutting process. A structured diamond tool with a groove orientation of $60^{\circ}$ produces a smaller cutting force, less cutting heat, and smaller von Mises stress and hydrostatic stress, but results in a larger frictional coefficient and more beta-silicon phase. Cutting with a smaller groove orientation causes slightly higher potential energy in subsurface. Furthermore, when the groove orientation was $\theta=0^{\circ}$, both the compressive normal stresses $\left(\sigma_{x x}, \sigma_{y y}\right)$ and the shear stress $\left(\tau_{x y}\right)$ were slightly larger, meaning that tools with a groove orientation of $0^{\circ}$ tend to cut silicon in a more ductile mode than other structured tools.

(2) Ground surface quality is strongly determined by groove depth, but the subsurface temperature is only slightly affected. A cutting tool with a smaller groove depth produced a slightly stronger material removal ability and tended to cut silicon in a more ductile mode. However, a groove depth of $0.8 \mathrm{~nm}$ resulted in smaller von Mises stress and a reduced cutting force.

(3) Smaller groove width tools tended to cut silicon in a more ductile mode, and improved material removal rate and ground surface quality. However, cutting with a smaller groove width tool caused more cutting heat and increased the resultant cutting force.

(4) Tools with larger groove factors led to a more ductile cutting mode and larger material removal rate. A groove factor of $\eta=40 \%$ reduced the overall magnitude of the resultant cutting forces. Furthermore, the average subsurface temperature increased with increasing groove factor, meaning that the groove on the tool accelerated heat dissipation in the subsurface atoms.

(5) Although the effect of groove shape on ground surface quality was very small during the nanoscale cutting process, cutting with a V-shaped groove can improve the material removal ability.

\section{Acknowledgements}

The authors would like to appreciate the support from the National Natural Science Foundation of China (No. 51675172).

\section{References}

1 J. Xie, Y. W. Zhuo and T. W. Tan, Experimental study on fabrication and evaluation of micro pyramid-structured silicon surface using a V-tip of diamond grinding wheel, Precis. Eng., 2011, 35(1), 173-182.

2 H. Angermann, J. Rappich, L. Korte, et al., Wet-chemical passivation of atomically flat and structured silicon substrates for solar cell application, Appl. Surf. Sci., 2008, 254(12), 3615-3625.

3 P. W. Butler-Smith, D. A. Axinte, M. Pacella, et al., Micro/ nanometric investigations of the effects of laser ablation in the generation of micro-tools from solid CVD diamond structures, J. Mater. Process. Technol., 2013, 213(2), 194-200.

4 J. Yan, M. Yoshino, T. Kuriagawa, T. Shirakashi, K. Syoji and R. Komanduri, On the ductile machining of silicon for micro 
electro-mechanical systems (MEMS), opto-electronic and optical applications, Mater. Sci. Eng., A, 2001, 297, 230-234.

5 F. Z. Fang, H. Wu and Y. C. Liu, Modelling and experimental investigation on nanometric cutting of monocrystalline silicon, Int. J. Mach. Tool. Manufact., 2005, 45, 1681-1686.

6 P. W. Butler-Smith, D. A. Axinte and M. Daines, Preferentially oriented diamond micro-arrays: a laser patterning technique and preliminary evaluation of their cutting forces and wear characteristics, Int. J. Mach. Tool. Manufact., 2009, 49, 1175-1184.

7 D. Dhupal, B. Doloi and B. Bhattacharyya, Pulsed Nd:YAG laser turning of micro-groove on aluminum oxide ceramic $\left(\mathrm{Al}_{2} \mathrm{O}_{3}\right)$, Int. J. Mach. Tool. Manufact., 2008, 48(2), 236-248.

8 B. Guo, Q. Zhao and X. Fang, Precision grinding of optical glass with laser micro-structured coarse-grained diamond wheels, J. Mater. Process. Technol., 2014, 214, 1045-1051.

9 C. Walter, T. Komischke, E. Weingärtner, et al., Structuring of CBN grinding tools by ultrashort pulse laser ablation, Procedia CIRP, 2014, 14, 31-36.

$10 \mathrm{~K}$. Zhou and B. Wei, Determination of the thermophysical properties of liquid and solid Ti-6Al-4V alloy, Appl. Phys. A: Mater. Sci. Process., 2016, 122(3), 1-5.

11 K. Zhou, H. P. Wang, J. Chang, et al., Experimental study of surface tension, specific heat and thermal diffusivity of liquid and solid titanium, Chem. Phys. Lett., 2015, 639, 105-108.

12 J. F. G. Oliveira, A. C. Bottene and T. V. Franca, A novel dressing technique for texturing of ground surfaces, CIRP Ann., 2010, 59(1), 361-364.

13 E. J. Silva, J. F. G. Oliveira, B. B. Salles, R. S. Cardoso and V. R. A. Reis, Strategies for production of parts textured by grinding using patterned wheels, CIRP Ann., 2013, 62(1), 355-358.

14 A. M. O. Mohamed, R. Bauer and A. Warkentin, Application of shallow circumferential grooved wheels to creep-feed grinding, J. Mater. Process. Technol., 2013, 213(5), 700-706.

15 H. Dai, G. Chen, C. Zhou, et al., A numerical study of ultraprecision machining of monocrystalline silicon with laser nano-structured diamond tools by atomistic simulation, Appl. Surf. Sci., 2017, 393, 405-416.

16 J. Gu, X. Yang, Z. Lv, et al., Functionalized graphite nanoplatelets/epoxy resin nanocomposites with high thermal conductivity, Int. J. Heat Mass Transfer, 2016, 92, 15-22.

17 J. Gu, C. Liang, J. Dang, et al., Fabrication of modified bismaleimide resins by hyperbranched phenyl polysiloxane and improvement of their thermal conductivities, $R S C$ Adv., 2016, 6(62), 57357-57362.

18 J. Gu, C. Liang, X. Zhao, et al., Highly thermally conductive flame-retardant epoxy nanocomposites with reduced ignitability and excellent electrical conductivities, Compos. Sci. Technol., 2017, 139, 83-89.

19 J. F. Belak and I. F. Stowers, A molecular dynamics model of the orthogonal cutting process, Lawrence Livermore National Lab, CA (USA), 1990, pp. 76-79.
20 R. Komanduri, N. Chandrasekaran and L. M. Raff, Effect of tool geometry in nanometric cutting: a molecular dynamics simulation approach, Wear, 1998, 219(1), 84-97.

$21 \mathrm{H}$. Dai and G. Chen, A molecular dynamics investigation into the mechanisms of material removal and subsurface damage of nanoscale high speed laser-assisted machining, Mol. Simul., 2016, 1-10.

22 C. Acevedo and A. Nussbaumer, Effect of tensile residual stresses on fatigue crack growth and S-N curves in tubular joints loaded in compression, Int. J. Fatigue, 2012, 36, 171180.

23 Y. Wang, J. Shi and C. Ji, A numerical study of residual stress induced in machined silicon surfaces by molecular dynamics simulation, Appl. Phys. A: Mater. Sci. Process., 2014, 115, 1263-1279.

$24 \mathrm{~J}$. Tersoff, Modeling solid-state chemistry: interatomic potentials for multicomponent systems, Phys. Rev. B: Condens. Matter Mater. Phys., 1989, 39, 5566.

25 S. Goel, X. Luo and R. L. Reuben, Wear mechanism of diamond tools against single crystal silicon in single point diamond turning process, Tribol. Int., 2013, 57, 272-281.

26 H. Dai, G. Chen, Q. Fang, et al., The effect of tool geometry on subsurface damage and material removal in nanometric cutting single-crystal silicon by a molecular dynamics simulation, Appl. Phys. A: Mater. Sci. Process., 2016, 122(9), 804.

27 Z. Tong, Y. Liang, X. Jiang, et al., An atomistic investigation on the mechanism of machining nanostructures when using single tip and multi-tip diamond tools, Appl. Surf. Sci., 2014, 290, 458-465.

28 Q. X. Pei, C. Lu and H. P. Lee, Large scale molecular dynamics study of nanometric machining of copper, Comput. Mater. Sci., 2007, 41(2), 177-185.

29 S. Goel, X. Luo, R. L. Reuben, et al., Influence of temperature and crystal orientation on tool wear during single point diamond turning of silicon, Wear, 2012, 284, 65-72.

30 J. Shi, C. Ji, Y. Wang, et al., Tool/Chip Interfacial Friction Analysis in Atomistic Machining of Polycrystalline Coppers, Journal of Micro and Nano-Manufacturing, 2014, 2(4), 041001.

31 Z. Tong, Y. Liang, X. Yang, et al., Investigation on the thermal effects during nanometric cutting process while using nanoscale diamond tools, Int. J. Adv. Manuf. Tech., 2014, 74(9-12), 1709-1718.

32 P. Zhu, Y. Hu, T. Ma, et al., Molecular dynamics study on friction due to ploughing and adhesion in nanometric scratching process, Tribol. Lett., 2011, 41, 41-46.

33 J. J. Zhang, T. Sun, Y. D. Yan, et al., Molecular dynamics simulation of subsurface deformed layers in AFM-based nanometric cutting process, Appl. Surf. Sci., 2008, 254, 4774-4779.

34 L. Zhang, H. Zhao, Y. Yang, et al., Evaluation of repeated single-point diamond turning on the deformation behavior of monocrystalline silicon via molecular dynamic simulations, Appl. Phys. A: Mater. Sci. Process., 2014, 116, 141-150. 
35 S. Plimpton, Fast parallel algorithms for short-range molecular dynamics, J. Comput. Phys., 1995, 117(1), 1-19.

36 A. Stukowski, Visualization and analysis of atomistic simulation data with OVITO-the Open Visualization Tool, Modell. Simul. Mater. Sci. Eng., 2010, 18, 015012.

37 S. Goel, A. Kovalchenko, A. Stukowski, et al., Influence of microstructure on the cutting behaviour of silicon, Acta Mater., 2016, 105, 464-478.

38 S. Goel, X. Luo, A. Agrawal, et al., Diamond machining of silicon: a review of advances in molecular dynamics simulation, Int. J. Mach. Tool. Manufact., 2015, 88, 131-164.

39 V. Domnich and Y. Gogotsi, Phase transformations in silicon under contact loading, Rev. Adv. Mater. Sci., 2002, 3(1), 1-36.

40 D. Ge, V. Domnich and Y. Gogotsi, High-resolution transmission electron microscopy study of metastable silicon phases produced by nanoindentation, J. Appl. Phys., 2003, 93(5), 2418-2423.

41 A. Kovalchenko, Y. Gogotsi, V. Domnich and A. Erdemir, Phase transformations in silicon under dry and lubricated sliding, Tribol. Trans., 2002, 45(3), 372-380.

42 S. Z. Chavoshi and X. Luo, Molecular dynamics simulation study of deformation mechanisms in 3C-SiC during nanometric cutting at elevated temperatures, Mater. Sci. Eng., A, 2016, 654, 400-417.

43 G. L. W. Cross, Silicon nanoparticles: isolation leads to change, Nat. Nanotechnol., 2011, 6, 467-468.
44 Q. H. Fang and L. C. Zhang, Prediction of the threshold load of dislocation emission in silicon during nanoscratching, Acta Mater., 2013, 61, 5469-5476.

45 D. E. Kim and S. I. Oh, Atomistic simulation of structural phase transformations in monocrystalline silicon induced by nanoindentation, Nanotechnology, 2006, 17(9), 2259.

46 Y. H. Lin and T. C. Chen, A molecular dynamics study of phase transformations in mono-crystalline $\mathrm{Si}$ under nanoindentation, Appl. Phys. A: Mater. Sci. Process., 2008, 92(3), 571-578.

47 L. Zhang, H. Zhao, Z. Ma, et al., A study on phase transformation of monocrystalline silicon due to ultraprecision polishing by molecular dynamics simulation, AIP Adv., 2012, 2, 042116.

48 Q. Tang and F. Chen, MD simulation of phase transformations due to nanoscale cutting on silicon monocrystals with diamond tip, J. Phys. D: Appl. Phys., 2006, 39, 3674.

49 D. Faken and H. Jónsson, Systematic analysis of local atomic structure combined with 3D computer graphics, Comput. Mater. Sci., 1994, 2, 279-286.

50 M. B. Cai, X. P. Li and M. Rahman, Study of the temperature and stress in nanoscale ductile mode cutting of silicon using molecular dynamics simulation, J. Mater. Process. Technol., 2007, 192, 607-612.

51 M. B. Cai, X. P. Li and M. Rahman, Study of the mechanism of nanoscale ductile mode cutting of silicon using molecular dynamics simulation, Int. J. Mach. Tool. Manufact., 2007, 47, 75-80. 